\title{
On triviality of S-matrix in conformal higher spin theory
}

\author{
Matteo Beccaria, ${ }^{a}$ Simon Nakach ${ }^{b}$ and Arkady A. Tseytlin ${ }^{b, 1}$ \\ ${ }^{a}$ Dipartimento di Matematica e Fisica Ennio De Giorgi, Università del Salento $G$ INFN, \\ Via Arnesano, 73100 Lecce, Italy \\ ${ }^{b}$ The Blackett Laboratory, Imperial College, \\ London $S W^{r}$ 2AZ, U.K. \\ E-mail: matteo.beccaria@le.infn.it, simon.nakach09@imperial.ac.uk, \\ tseytlin@imperial.ac.uk
}

ABSTRACT: We consider the conformal higher spin (CHS) theory in $d=4$ that contains the $s=1$ Maxwell vector, $s=2$ Weyl graviton and their higher spin $s=3,4, \ldots$ counterparts with higher-derivative $\square^{s}$ kinetic terms. The interacting action for such theory can be found as the coefficient of the logarithmically divergent part in the induced action for sources coupled to higher spin currents in a free complex scalar field model. We explicitly determine some cubic and quartic interaction vertices in the CHS action from scalar loop integrals. We then compute the simplest tree-level 4 -particle scattering amplitudes $11 \rightarrow 11$, $22 \rightarrow 22$ and $11 \rightarrow 22$ and find that after summing up all the intermediate CHS exchanges they vanish. This generalises the vanishing of the scattering amplitude for external conformal scalars interacting via the exchange of all CHS fields found earlier in arXiv:1512.08896. This vanishing should generalise to all scattering amplitudes in the CHS theory and as in the conformal scalar scattering case should be a consequence of the underlying infinite dimensional higher spin symmetry that extends the standard conformal symmetry.

Keywords: Higher Spin Gravity, Conformal and W Symmetry, Higher Spin Symmetry

ArXiv ePrint: 1607.06379

\footnotetext{
${ }^{1}$ Also at Lebedev Institute, Moscow, Russia.
} 


\section{Contents}

1 Introduction 1

2 Vertices in induced conformal higher spin action 5

3 Scattering in CHS theory: 4-vector amplitude 9

$\begin{array}{ll}3.14 \text {-vector exchange amplitude } & 10\end{array}$

$\begin{array}{lll}3.2 & \text { Summing over spins } & 13\end{array}$

4 General structure of CHS exchange amplitudes $\quad 14$

$\begin{array}{lll}5 & \text { Scattering amplitudes with conformal gravitons } & 17\end{array}$

$\begin{array}{lll}5.122 \rightarrow 22 \text { scattering } & 17\end{array}$

$5.2 \quad 11 \rightarrow 22$ scattering $\quad 20$

6 Concluding remarks $\quad 23$

A Vertices in CHS action from scalar loop integrals $\quad 24$

B Vanishing of 4-particle amplitude at special kinematics $\quad 27$

B.1 $11 \rightarrow 11$ scattering $\quad 27$

$\begin{array}{lll}\text { B.2 } \mathrm{j} \mathrm{j} \rightarrow \mathrm{j} \text { j scattering } & 27\end{array}$

C Derivation of the general form of the $11 \rightarrow 11$ spin $s$ exchange amplitude 29

\section{Introduction}

Conformal higher spin (CHS) theory [1-7] is a remarkable example of a formally consistent (gauge-invariant, though higher-derivative and thus non-unitary) higher spin model that has a local action with a flat-space vacuum and one dimensionless coupling constant.

It is naturally associated with another consistent higher spin theory, i.e. 2-derivative massless higher spin theory in AdS space of one dimension higher. Starting with a free complex scalar equation in 4 dimensions ${ }^{1} \partial^{2} \varphi=0$ one gets an infinite tower of conserved traceless totally symmetric higher spin currents $J_{s}=\varphi^{*} \mathcal{J}_{s} \varphi, \mathcal{J}_{s} \sim \partial^{s}+\ldots, s=1,2, \ldots, \infty$ that generalize the spin 0 primary operator $J_{0}=\varphi^{*} \varphi$. The conserved charges generate an infinite dimensional symmetry algebra of the free scalar equation $[8,9]$ that is associated to a collection of conformal Killing tensors. The CHS theory may be viewed as a gauge theory of this higher spin global symmetry. A closely related approach is based on interpreting

\footnotetext{
${ }^{1}$ In this paper we shall concentrate on the $d=4$ case but most of our discussion may be generalised to even $d>4$.
} 
CHS fields as "sources" for the currents $J_{s}$ that then inherit the linearised gauge invariances $\delta h_{\mu_{1} \ldots \mu_{s}}=\partial_{\left(\mu_{1}\right.} \epsilon_{\left.\mu_{2} \ldots \mu_{s}\right)}+\eta_{\left(\mu_{1} \mu_{2}\right.} \alpha_{\left.\mu_{3} \ldots \mu_{s}\right)}$ which generalise the usual reparametrisations and Weyl symmetry of the conformal gravity. Starting with free $\mathrm{U}(N)$ complex scalar CFT, adding source terms $h_{s} J_{s}$ to the free action $\partial \varphi^{*} \partial \varphi$ and integrating over $\varphi$ one finds for the generating functional of correlators of the currents $J_{s}$

$$
\Gamma[h]=N \log \operatorname{det}\left(\partial^{2}+\sum_{s} h_{s} \mathcal{J}_{s}\right), \quad \mathcal{J}_{s} \sim \partial^{s} .
$$

From the vectorial AdS/CFT point of view [10] the $4 \mathrm{~d}$ currents $J_{s}$ are dual to massless higher spins in $\mathrm{AdS}_{5}$ and the generating functional $\Gamma[h]$ should then be equal to the on-shell value of the AdS action with $h_{s}$ being the boundary values of the higher spin fields in AdS.

One can then obtain a local gauge-invariant action for the CHS fields $h_{s}$ by identifying it with the logarithmically UV divergent part of the "induced" action (1.1) [3-5]

$$
\begin{aligned}
S[h] & \left.\sim \log \operatorname{det}\left(\partial^{2}+\sum_{s} h_{s} \mathcal{J}_{s}\right)\right|_{\log \Lambda} \\
& \sim \frac{1}{g^{2}} \sum_{s} \int d^{4} x\left(h_{s} \partial^{2 s} h_{s}+\partial^{s_{1}+s_{2}+s_{3}-2} h_{s_{1}} h_{s_{2}} h_{s_{3}}+\partial^{s_{1}+s_{2}+s_{3}+s_{4}-4} h_{s_{1}} h_{s_{2}} h_{s_{3}} h_{s_{4}}+\ldots\right) .
\end{aligned}
$$

Here we introduced an arbitrary dimensionless coupling constant $g$ and indicated symbolically the overall powers of derivatives in the kinetic and interaction terms that follow from dimensional analysis. Indeed, as the $4 \mathrm{~d}$ scalar $\varphi$ has mass dimension 1 , the current $J_{s}$ has dimension $2+s$ and thus the corresponding source field $h_{s}$ must have the "shadow"-field dimension $\Delta_{s}=2-s$ (i.e. 1 for vector field, 0 for conformal graviton, etc.). This then determines the derivative structure of (1.2). ${ }^{2}$ In particular, the presence of $2 s$ derivatives in the kinetic term in (1.2) is consistent with both the above linearised gauge invariance $\delta h_{s}=\partial \epsilon_{s-1}+\eta_{2} \alpha_{s-2}$ and the locality of the action. ${ }^{3}$

In addition to the linearised gauge symmetry and the standard conformal symmetry the CHS action should be invariant under the full infinite dimensional CHS symmetry [4] whose global part is the symmetry of free scalar $4 \mathrm{~d}$ Laplace equation. This large symmetry should provide strong constraints on the corresponding classical and quantum theory. For example, for fixed spectrum of the CHS fields the action (1.2) should be essentially unique (modulo field redefinitions) ${ }^{4}$ and thus renormalizable. In view of the conformal symmetry being gauged here it should actually be UV finite, provided the theory is quantum-consistent, i.e. there are no conformal and higher symmetry anomalies. An indication of a hidden simplicity of the CHS theory is the vanishing of the regularised total number of its degrees

\footnotetext{
${ }^{2}$ The fact that the powers of derivatives are directly correlated with the values of the spins in the vertex (which is a consequence of the underlying conformal invariance) is an important simplifying feature of this theory compared to the AdS higher spin theory and a hypothetic 2-derivative massless higher spin theory in flat space that both contain a dimensional parameter.

${ }^{3}$ The kinetic term should contain the transverse traceless spin $s$ projector $\Pi_{s}$ that is given by products of $s$ factors of $\Pi_{\nu}^{\mu}=\delta_{\nu}^{\mu}-\frac{\partial^{\mu} \partial_{\nu}}{\partial^{2}}$ and thus $\Pi_{s} \partial^{2 s}$ is local.

${ }^{4}$ Starting instead from a free scalar CFT with a free spinor or free Maxwell vector CFT one gets a different spectrum of conserved currents and thus a different "induced" CHS theory.
} 
of freedom, or, equivalently, the triviality of the free (one-loop) partition function in flat space [11]. This partition function vanishes also on 4 -sphere implying the vanishing of the Weyl anomaly a-coefficient $[12,13]$ (which is also in agreement with a triviality of the 1-loop correction to the massless HS partition function as required by the AdS/CFT [14]). ${ }^{5}$ Similar vanishing was also found (under some natural assumptions) for the 1-loop Weyl anomaly $c$-coefficient [11, 13-15]. As the Weyl symmetry is one of the CHS gauge symmetries, the same anomaly cancellation may apply also to all algebraic CHS gauge symmetries.

The global part of the CHS symmetry should also strongly constrain other "observables", e.g., the analog of the S-matrix involving exchanges of the CHS fields. Indeed, it was found in [16] that starting with a free external scalar field coupled (via the above current $\int d^{4} x h_{s} J_{s}$ interaction) to free CHS fields with the action $\int d^{4} x \sum_{s} h_{s} \Pi_{s} \partial^{2 s} h_{s}$ and computing the 4-scalar tree level scattering due to the exchange of the tower of CHS fields one finds that while the individual spin $s$ exchange contributions are non-trivial, their sum over all $s=0,1,2, \ldots$ vanishes. This vanishing can be understood [16] as a consequence of the CHS global symmetry of the coupled theory (in particular, the "hypertranslations" $\delta \varphi=\epsilon^{\mu_{1} \ldots \mu_{s}} \partial_{\mu_{1}} \ldots \partial_{\mu_{s}} \varphi$ and scale invariance).

The aim of the present paper is to show that this triviality of the 4-particle scattering amplitude is found also when the external scalars are replaced by the CHS fields themselves with cubic and quartic interactions given by (1.2). We shall consider few particular examples of the CHS 4-particle scattering amplitudes (4-vector, 4-graviton, etc.) and find that after summation over all exchanged conformal higher spins the total amplitude vanishes. This cancellation is rather non-trivial and like in the external scalar amplitude case [16] should again be a consequence of the underlying higher spin global symmetry of the theory (and should thus be a manifestation of a "generalised" Coleman-Mandula theorem). This suggests that the full "S-matrix" of the CHS theory should be trivial. ${ }^{6}$

To be able to compute scattering amplitudes of CHS states one needs first to determine the precise structure of vertices in the "induced" action (1.2). For that one needs to find the logarithmically divergent (or $\frac{1}{\varepsilon}$ pole in dimensional regularisation) terms in the one-loop scalar loop diagrams with the two, three or four current $J_{s}$ insertions. We shall choose the external $h_{s}$ legs to be in the transverse traceless gauge. ${ }^{7}$ Having found the relevant terms in the action (1.2) we will define the 4-particle scattering S-matrix as the amputated tree-level Green's function (i.e. the sum of the exchange term and contact vertex $>-<<+><$ ) contracted with particular on-shell asymptotic states. For $s=1$ vector the asymptotic states are the standard helicity \pm 1 states, while in the $s>1$ case with the free equation (in TT gauge) $\partial^{2 s} h_{s}=0$ describing total of $s(s+1)$ dynamical degrees of freedom one may choose a special solution corresponding, e.g., to the standard massless helicity $\pm s$ field. ${ }^{8}$

\footnotetext{
${ }^{5}$ The definition of the sum over spins requires a particular prescription that should be consistent with the underlying symmetries $[11,15]$.

${ }^{6}$ As we are dealing with a non-unitary higher derivative theory containing an infinite number of fields some assumptions of the standard Coleman-Mandula theorem may not directly apply. In particular, the definition of the scattering matrix for higher-derivative fields requires clarification, see below.

${ }^{7}$ This avoids, in particular, the explicit discussion of field redefinitions eliminating the traces.

${ }^{8}$ For example, in the case of the Weyl graviton one can always solve the linearised Bach equations by imposing the linearised Einstein equations. One may also consider other special choices of solutions of $\partial^{4}$ equations as asymptotic states.
} 
Before turning to the discussion of higher spin terms in (1.2) let us first recall the structure of the non-linear terms for the low $(s=0,1,2$,$) spins only. Instead of starting$ with the scalar action involving only the linear coupling to the background fields $h_{0}, h_{1}, h_{2}$ (which here we assume to be subject to TT condition and drop total derivatives)

$$
L=-\partial_{\mu} \varphi^{*} \partial^{\mu} \varphi+\sum_{s} h_{s} \varphi^{*} \mathcal{J}_{s} \varphi=\partial_{\mu} \varphi^{*} \partial^{\mu} \varphi+h_{0} \varphi^{*} \varphi+i h^{\mu} \varphi^{*} \partial_{\mu} \varphi+\frac{1}{2} h^{\mu \nu} \partial_{\mu} \varphi^{*} \partial_{\nu} \varphi+\ldots
$$

let us start with the standard manifestly (U(1), reparametrization and Weyl) covariant coupling of a complex scalar field to the background metric $g_{\mu \nu}=\eta_{\mu \nu}+h_{\mu \nu}^{\prime}$, vector field $h_{\mu}^{\prime}$ and a scalar $h_{0}^{\prime}$, i.e.

$$
I=\int d^{4} x \sqrt{g}\left[-g^{\mu \nu} D_{\mu} \varphi^{*} D_{\nu} \varphi+\left(h_{0}^{\prime}-\frac{1}{6} R\right) \varphi^{*} \varphi\right], \quad D_{\mu} \varphi=\partial_{\mu} \varphi+\frac{i}{2} h_{\mu}^{\prime} \varphi .
$$

The log UV divergent part of the resulting scalar determinant (cf. (1.1)) is given by the standard covariant Seeley coefficient expression (we ignore unimportant overall constant related to coupling $g$ in $(1.2))^{9}$

$$
S\left[h_{0}^{\prime}, h_{1}^{\prime}, h_{2}^{\prime}\right]=\int d^{4} x \sqrt{g}\left(h_{0}^{\prime 2}-\frac{1}{24} F_{\mu \nu}^{\prime 2}+\frac{1}{60} C_{\mu \nu \lambda \rho}^{2}\right),
$$

where $F_{\mu \nu}^{\prime}=\partial_{\mu} h_{\nu}^{\prime}-\partial_{\nu} h_{\mu}^{\prime}$ and $C$ is the Weyl tensor for $g_{\mu \nu}$. Since the fields $h_{s}^{\prime}$ in (1.4) are related to $h_{s}$ in (1.3) by a local non-linear redefinition

$$
\begin{aligned}
& h_{0}^{\prime}=h_{0}+\frac{1}{4} h_{\mu} h^{\mu}+\frac{1}{96}\left(\partial_{\lambda} h_{\mu \nu} \partial^{\lambda} h^{\mu \nu}+2 h_{\mu \nu} \square h^{\mu \nu}+2 \partial_{\lambda} h_{\mu \nu} \partial^{\mu} h^{\lambda \nu}\right)+\ldots, \\
& h_{\mu}^{\prime}=h_{\mu}+\frac{1}{2} h_{\mu \nu} h^{\nu}+\frac{1}{4} h_{\mu \nu} h^{\nu \lambda} h_{\lambda}+\ldots, \quad h_{\mu \nu}^{\prime}=\frac{1}{2} h_{\mu \nu}+\frac{1}{4} h_{\mu \lambda} h_{\nu}^{\lambda}-\frac{1}{16} \eta_{\mu \nu} h^{\lambda \rho} h_{\lambda \rho}+\ldots,
\end{aligned}
$$

expanding (1.5) we may thus read off the cubic and quartic couplings of the original $h_{0}, h_{\mu}, h_{\mu \nu}$ fields in (1.2). In particular, using (1.6) we find that the scalar-vector sector of (1.5) takes the form

$$
S\left[h_{0}, h_{1}\right]=\int d^{4} x\left[\left(h_{0}+\frac{1}{4} h_{\mu} h^{\mu}\right)^{2}-\frac{1}{24} F_{\mu \nu}^{2}\right]
$$

Thus the simplest cubic and quartic vertices are 011 and 1111. We also conclude, in particular, that the contribution of the $h_{0}$ exchange to the 4 -vector scattering amplitude cancels against the 4 -vector contact vertex. As there is no 3 -vector coupling, the full 4 -vector tree-level amplitude should thus be given by the sum of all exchanges of CHS fields with $s \geq 2$ and happens to vanish as we will find in section 3. Similarly, the 112 vertex is related to the one in the Maxwell-Weyl theory, the 222 and 2222 vertices are related to those in the Weyl theory, etc. Thus the contribution to the 4-graviton amplitude computed from the $s=0,2$ exchanges and the 2222 vertex should be the same as the

\footnotetext{
${ }^{9}$ Note that this action may be interpreted as the bosonic sector of $\mathcal{N}=1$ conformal supergravity action with $h_{0}^{\prime}$ playing the role of the auxiliary field.
} 
4-graviton amplitude in pure Weyl theory (that happens to vanish). The contributions of all $s>2$ CHS exchanges vanishes separately as we shall demonstrate in section 5 .

This paper is organised as follows. In section 2 we shall present the results for some cubic CHS vertices (relevant for the computation of spin 1 and spin 2 scattering amplitudes below) from the UV singular parts of the scalar loop integrals, with some details relegated to appendix A. In section 3 we shall compute the 4-vector scattering amplitude and demonstrate that after summing over all CHS exchanges it vanishes. We shall then observe in section 4 that the conformal higher spin exchange amplitudes should have the same general structure as the partial wave amplitudes in the representation of [17]. In section 5 we shall find that the scattering amplitudes $22 \rightarrow 22$ and $11 \rightarrow 22$ involving conformal gravitons do have this expected structure and they also vanish once one sums up all intermediate CHS exchanges. Some concluding remarks will be made in section 6 . In appendix B we shall independently verify the vanishing of the $11 \rightarrow 11$ amplitude at special kinematics (backward scattering) and find that this vanishing appears to generalise to the case of $\mathrm{jj} \rightarrow \mathrm{jj}$ scattering with all $\mathrm{j}=1,2,3, \ldots$ supporting our conjecture that the full 4-particle S-matrix in CHS theory should be trivial. In appendix $\mathrm{C}$ we shall give the general derivation of the expression for the CHS spin $s$ exchange contribution to the $11 \rightarrow 11$ amplitude.

\section{Vertices in induced conformal higher spin action}

To be able to compute the CHS scattering amplitudes we should first determine the relevant cubic and quartic terms in the "induced" action (1.2). We shall use the following notation for totally symmetric tensors: $J_{\mu(s)} \equiv J_{\mu_{1} \ldots \mu_{s}}$ and also $\partial_{\mu(s)} \equiv \partial_{\mu_{1}} \ldots \partial_{\mu_{s}}$. Our starting point will be the complex scalar Lagrangian in external CHS background (see, e.g., [5, 16] and refs. there)

$$
\begin{aligned}
\mathscr{L} & =-\partial_{\mu} \varphi^{*} \partial^{\mu} \varphi+\sum_{s=0}^{\infty} J_{\mu(s)} h^{\mu(s)}, \\
J_{\mu(s)}(x) & =\frac{i^{s} 2^{s} s !}{(2 s) !} \sum_{k=0}^{s}\left(\begin{array}{c}
s \\
k
\end{array}\right)\left(\begin{array}{c}
\frac{s+k-1}{2} \\
s
\end{array}\right) G_{\mu(s)}^{(k)}, \\
G_{\mu(s)}^{(k)} & =\left[\left(\partial-\partial^{\prime}\right)_{\mu(k)}\left(\partial+\partial^{\prime}\right)_{\mu(s-k)} \varphi(x) \varphi^{*}\left(x^{\prime}\right)\right]_{x=x^{\prime}},
\end{aligned}
$$

where the low-spin currents $J_{\mu(s)}$ are

$$
\begin{aligned}
J & =\varphi \varphi^{*}, \quad J_{\mu}=\frac{i}{2}\left(\varphi^{*} \partial_{\mu} \varphi-\varphi \partial_{\mu} \varphi^{*}\right), \\
J_{\mu \nu} & =\frac{1}{6}\left[\partial_{\mu} \varphi \partial_{\nu} \varphi^{*}+\partial_{\nu} \varphi \partial_{\mu} \varphi^{*}-\frac{1}{2}\left(\varphi^{*} \partial_{\mu} \partial_{\nu} \varphi+\varphi \partial_{\mu} \partial_{\nu} \varphi^{*}\right)\right] .
\end{aligned}
$$

The vertices in the CHS action (1.2) may be thought of as originating from the coincidingpoint limits of the current correlators in the free scalar CFT $\left.\left\langle J_{s_{1}}\left(x_{1}\right) \ldots J_{s_{n}}\left(x_{n}\right)\right\rangle\right|_{x_{i} \rightarrow x}$ and can be found in coordinate space using, e.g., differential regularization [18]. Here we shall use momentum space representation and dimensional regularisation $(d=4-\varepsilon)$ and define 
the classical CHS action $S[h]$ as in (1.2), i.e. as the UV pole part of the one-loop scalar $\varphi$ effective action:

$$
\Gamma[h]=\frac{1}{(4 \pi)^{2} \varepsilon} S[h]+\text { finite } .
$$

In general, the CHS action should contain an arbitrary dimensionless constant $g$ as in (1.2) that will then appear as $g^{2}$ factor in the resulting 4-particle tree-level amplitude; in what follows we shall ignore this universal overall factor, i.e. set $g=1$.

We shall also assume that the background fields $h_{s} \equiv h_{\mu(s)}$ in (2.1) are transverse and traceless (TT) as this will be sufficient for the subsequent computation of the on-shell scattering amplitudes. ${ }^{10}$ Note that in this case we may integrate by parts to write the interaction terms as $h^{\mu} J_{\mu} \rightarrow i h^{\mu} \varphi^{*} \partial_{\mu} \varphi, \quad h^{\mu \nu} J_{\mu \nu} \rightarrow \frac{1}{2} h^{\mu \nu} \partial_{\mu} \varphi^{*} \partial_{\nu} \varphi$ or $-\frac{1}{2} h^{\mu \nu} \varphi^{*} \partial_{\mu} \partial_{\nu} \varphi$, etc. In general, for TT fields the $h_{s}\left(p^{\prime}-p\right) \varphi^{*}\left(-p^{\prime}\right) \varphi(p)$ vertex in momentum representation reduces simply to

$$
V_{\mu(s)}(p)=\frac{1}{s !} p_{\mu_{1}} \cdots p_{\mu_{s}},
$$

where $p$ is momentum of the $\varphi$ leg. We can then compute the UV singular part of the scalar loop diagram with two $V_{\mu(s)}$ insertions

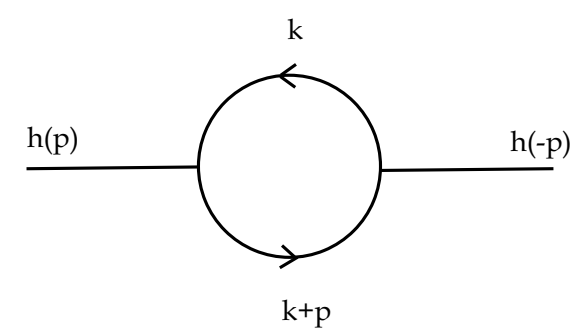

We then find that the kinetic term in the CHS action (restricted to TT fields) is given by ${ }^{11}$

$$
S_{2}[h]=\sum_{s=0,1,2, \ldots} \frac{1}{2^{s}(2 s+1) !} \int d^{4} x h_{\mu(s)} \square^{s} h^{\mu(s)} .
$$

To determine the cubic $h_{s_{1}} h_{s_{2}} h_{s_{3}}$ couplings in the CHS action (1.2) we are to compute the UV singular part of the one-loop scalar diagram with three (spin $s_{1}, s_{2}$ and $s_{3}$ ) current vertex (2.6) insertions

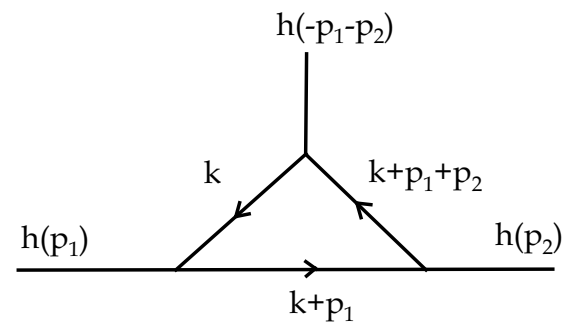

\footnotetext{
${ }^{10}$ In contrast to usual massless Fronsdal HS fields (where one can only fix transverse or de Donder gauge off shell) for the conformal higher spin fields the gauge symmetry involves both the differential and the algebraic symmetry allowing one to fix TT gauge, and this leads to substantial simplifications.

${ }^{11}$ The relative normalisations of the first $s=0,1,2$ terms here are the same as in (1.5), (1.6): note that $h_{\mu \nu}^{\prime}=g_{\mu \nu}-\eta_{\mu \nu}$ in the manifestly covariant action (1.4) is given by $h_{\mu \nu}^{\prime}=\frac{1}{2} h_{\mu \nu}+\ldots$ in terms of $h_{\mu \nu}$ in (2.1) so that $C_{\mu \nu \lambda \rho}^{2} \rightarrow 2 R_{\mu \nu}^{2}+\ldots \rightarrow \frac{1}{2} h^{\prime \mu \nu} \square^{2} h_{\mu \nu}^{\prime} \rightarrow \frac{1}{8} h^{\mu \nu} \square^{2} h_{\mu \nu}$.
} 
As each spin $s$ vertex involves $s$ derivatives, the parity invariance implies that the resulting interaction is non-zero only if $s_{1}+s_{2}+s_{3}=$ even. One can also show (using that $h_{s}$ is subject to the TT condition and dimensional analysis) that $0-0-s$ interaction vanishes for all $s$, i.e. $S_{3}\left[h_{0}, h_{0}, h_{s}\right]=0$.

For $s_{1}=s_{2}=1$ the interaction 1-1-s is non-zero only if $s$ is even. Written in coordinate space the corresponding cubic interaction in the CHS action (1.2) (restricted again to TT fields) is found to be (see appendix A) ${ }^{12}$

$$
\begin{aligned}
& S_{3}\left[h_{1}, h_{1}, h_{s}\right]=\frac{(-1)^{s / 2}}{(s+2) !} \int d^{4} x\left[\partial_{\rho(s)} h_{\mu} h^{\mu} h^{\rho(s)}-2 h_{\mu} \partial^{\mu} \partial_{\rho(s-1)} h_{\nu} h^{\nu \rho(s-1)}\right. \\
& \left.-\partial_{\lambda} \partial^{\rho(s-2)} h^{\mu} \partial^{\lambda} h^{\nu} h_{\mu \nu \rho(s-2)}-\frac{s}{2} \partial^{\rho(s-2)} \square h^{\mu} h^{\nu} h_{\mu \nu \rho(s-2)}-\frac{s}{2} \partial^{\rho(s-2)} h^{\mu} \square h^{\nu} h_{\mu \nu \rho(s-2)}\right] .
\end{aligned}
$$

This vertex has total of $s$ derivatives in agreement with the general structure of the CHS action (1.2). In particular,

$$
S_{3}\left[h_{1}, h_{1}, h_{0}\right]=\frac{1}{2} \int d^{4} x h_{\mu} h^{\mu} h_{0}
$$

is in agreement with (1.5), (1.7). We may also compute the 4-vector quartic vertex from the UV pole part of the diagram

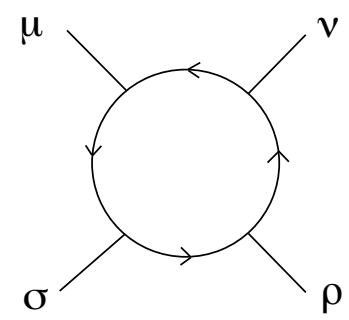

getting, in agreement with (1.7),

$$
S_{4}\left[h_{1}, h_{1}, h_{1}, h_{1}\right]=\frac{1}{16} \int d^{4} x\left(h_{\mu} h^{\mu}\right)^{2} .
$$

The vector-vector-graviton coupling in (2.10)

$$
S_{3}\left[h_{1}, h_{1}, h_{2}\right]=\frac{1}{24} \int d^{4} x\left[\partial_{\rho} h_{\mu} \partial_{\sigma} h^{\mu} h^{\rho \sigma}-2 \partial_{\rho} h_{\mu} \partial^{\mu} h_{\nu} h^{\nu \rho}+\partial_{\rho} h^{\mu} \partial^{\rho} h^{\nu} h_{\mu \nu}+2 h^{\mu} \square h^{\nu} h_{\mu \nu}\right]
$$

is equivalent (for TT fields) to the standard graviton-Maxwell coupling in (1.5) provided one takes into account the redefinitions in (1.6).

Similar expressions are found when the vector vertices in (2.9) are replaced by the graviton ones, i.e. for the case of $s_{1}=s_{2}=2, s_{3}=s$ interaction term (see appendix A). With $p_{1}, p_{2}$ being spin 2 momenta the resulting 2-2-s interaction vertex contains $s+2$ powers of momentum and reads $\mathrm{V}_{\mu_{1} \mu_{2}, \nu_{1} \nu_{2}, \rho(s)}=V_{\mu_{1} \mu_{2}, \nu_{1} \nu_{2}, \rho(s)}\left(p_{1}, p_{2}\right)+V_{\mu_{1} \mu_{2}, \nu_{1} \nu_{2}, \rho(s)}\left(p_{2}, p_{1}\right)$,

\footnotetext{
${ }^{12}$ The last two terms involving $\square h^{\mu}$, i.e. proportional to the vector field equation of motion can be, in principle, redefined away.
} 
where $^{13}$

$$
\begin{aligned}
& V_{\mu_{1} \mu_{2}, \nu_{1} \nu_{2}, \rho(s)}\left(p_{1}, p_{2}\right)=\frac{1}{8(s+4) !}\left[-\sum_{\mu \neq \mu^{\prime}, \nu \neq \nu^{\prime}} \eta_{\mu \nu} p_{2 \mu^{\prime}} p_{1 \nu^{\prime}}\left(p_{1}\right)_{\rho(s)}\right. \\
& \left.\quad+2 \sum_{\mu \neq \mu^{\prime}} p_{2 \mu^{\prime}} p_{1 \nu_{1}} p_{1 \nu_{2}} \eta_{\mu \rho_{1}} p_{1 \rho_{2}} \ldots p_{1 \rho_{s}}-2 \sum_{\nu \neq \nu^{\prime}} p_{1 \nu^{\prime}} p_{2 \mu_{1}} p_{2 \mu_{2}} \eta_{\nu \rho_{1}} p_{1 \rho_{2}} \ldots p_{1 \rho_{s}}\right] \\
& \quad \frac{p_{1} \cdot p_{2}}{16(s+4) !}\left\{2\left(\eta_{\mu_{1} \nu_{1}} \eta_{\mu_{2} \nu_{2}}+\eta_{\mu_{1} \nu_{2}} \eta_{\mu_{2} \nu_{1}}\right)\left(p_{1}\right)_{\rho(s)}\right. \\
& \quad-4\left(p_{1 \nu_{1}} \eta_{\mu_{1} \nu_{2}} \eta_{\mu_{2} \rho_{1}}-p_{2 \mu_{1}} \eta_{\mu_{2} \nu_{1}} \eta_{\nu_{2} \rho_{1}}+\operatorname{sym} \mu_{1,2}, \nu_{1,2}\right) p_{1 \rho_{2}} \cdots p_{1 \rho_{s}} \\
& +\left[6\left(\eta_{\mu_{1} \rho_{1}} \eta_{\mu_{2} \rho_{2}}+\eta_{\mu_{1} \rho_{2}} \eta_{\mu_{2} \rho_{1}}\right) p_{1 \nu_{1}} p_{1 \nu_{2}}+6\left(\eta_{\nu_{1} \rho_{1}} \eta_{\nu_{2} \rho_{2}}+\eta_{\nu_{1} \rho_{2}} \eta_{\nu_{2} \rho_{1}}\right) p_{2 \mu_{1}} p_{2 \mu_{2}}\right. \\
& \left.\quad-\sum_{\mu \neq \mu^{\prime}, \nu \neq \nu^{\prime}} \sum_{\left.\left.\left(\eta_{\mu \rho_{1}} \eta_{\nu \rho_{2}}+\eta_{\mu \rho_{2}} \eta_{\nu \rho_{1}}\right) p_{2 \mu^{\prime}} p_{1 \nu^{\prime}}\right] p_{1 \rho_{3}} \cdots p_{1 \rho_{s}}\right\}}\right\} \\
& +\frac{\left(p_{1} \cdot p_{2}\right)^{2}}{8(s+4) !} \sum_{\mu \neq \mu^{\prime}, \nu \neq \nu^{\prime}}\left(\eta_{\mu \rho_{1}} \eta_{\nu \rho_{2}} \eta_{\mu^{\prime} \nu^{\prime}}+\eta_{\mu \rho_{2}} \eta_{\nu \rho_{1}} \eta_{\mu^{\prime} \nu^{\prime}}\right) p_{1 \rho_{3}} \cdots p_{1 \rho_{s}} \\
& \left.\quad-\left(p_{1 \nu_{1}} \eta_{\mu_{1} \rho_{1}} \eta_{\mu_{2} \rho_{2}} \eta_{\nu_{2} \rho_{3}}-p_{2 \mu_{1}} \eta_{\mu_{2} \rho_{1}} \eta_{\nu_{1} \rho_{2}} \eta_{\nu_{2} \rho_{3}}+\operatorname{sym}_{1,2,3}\right) p_{1 \rho_{4}} \cdots p_{1 \rho_{s}}\right\} \\
& \quad \frac{\left(p_{1} \cdot p_{2}\right)^{3}}{32(s+4) !}\left(\eta_{\mu_{1} \rho_{1}} \eta_{\mu_{2} \rho_{2}} \eta_{\nu_{1} \rho_{3}} \eta_{\nu_{2} \rho_{4}}+\operatorname{sym} \rho_{1,2,3,4}\right) p_{1 \rho_{5}} \cdots p_{1 \rho_{s}},
\end{aligned}
$$

where sym stands for adding terms ensuring symmetry in $\left(\mu_{1}, \mu_{2}\right),\left(\nu_{1}, \nu_{2}\right)$ and $\left(\rho_{1}, \ldots, \rho_{s}\right)$. In particular, choosing $s=0$ we find that the 2-2-0 coupling term in the CHS action can be written as

$$
S_{3}\left[h_{0}, h_{2}, h_{2}\right]=\frac{1}{48} \int d^{4} x h_{0}\left(\partial_{\rho} h_{\mu \nu} \partial^{\rho} h^{\mu \nu}+2 \partial_{\rho} h_{\mu \nu} \partial^{\mu} h^{\rho \nu}\right) .
$$

One can trace the origin of this term to $h_{0}^{\prime 2}$ term in (1.5) and the redefinition (1.6) (in particular, it corresponds to cross-term $h_{0} R$ with $R$ in (1.4) expanded to quadratic order in $h_{\mu \nu}^{\prime}$ ).

The 1-0-s vertex multiplying $h_{\mu}\left(p_{1}\right), h_{0}\left(p_{2}\right)$ and $h_{\rho(s)}\left(-p_{1}-p_{2}\right)$ is non-zero when $s$ is odd and is found to be (where symmetrisation in $\rho_{i}$ is assumed)

$$
\mathrm{V}_{\mu, \rho(s)}=\frac{2}{(s+1) !} \eta_{\mu \rho_{1}} p_{\rho_{2}} \cdots p_{\rho_{s}} .
$$

Here $p$ stands for either $p_{1}$ or $p_{2}\left((2.17)\right.$ is symmetric under $p_{1} \rightarrow p_{2}$ as the fields are assumed to be TT and $s$ is odd). Similarly, the 2-0-s vertex (non-vanishing for $s=$ even) is

\footnotetext{
${ }^{13}$ Here we drop terms proportional to equations of motion for spin 2 states as we will be using this vertex to compute 2-2-2-2 scattering amplitude. Note, however, that for spin 2 exchange one is to use the 2-2-2 vertex that is symmetric in the three spin 2 legs with no on-shell condition assumed.
} 
given by

$$
\begin{aligned}
\mathrm{V}_{\mu_{1} \mu_{2}, \rho(s)}=\frac{1}{(s+2) !} & {\left[-\left(\eta_{\mu_{1} \rho_{1}} p_{1 \mu_{2}}+\eta_{\mu_{2} \rho_{1}} p_{1 \mu_{1}}\right) p_{1 \rho_{2}} \ldots p_{1 \rho_{s}}\right.} \\
& \left.-\frac{1}{2} p_{1} \cdot p_{2}\left(\eta_{\mu_{1} \rho_{1}} \eta_{\mu_{2} \rho_{2}}+\eta_{\mu_{2} \rho_{2}} \eta_{\mu_{1} \rho_{1}}\right) p_{1 \rho_{3}} \ldots p_{1 \rho_{s}}\right] .
\end{aligned}
$$

In the case of 1-2-s vertex (with $s=$ odd) appearing multiplied by the TT fields $h_{\mu_{1} \mu_{2}}\left(p_{1}\right)$, $h_{\nu}\left(p_{2}\right), h_{\rho(s)}\left(-p_{1}-p_{2}\right)$ we get

$$
\begin{aligned}
& \mathrm{V}_{\mu_{1} \mu_{2}, \nu, \rho(s)}\left(p_{1}, p_{2}\right)=\frac{1}{(s+3) !}\left\{\left(\eta_{\mu_{1} \nu} p_{2 \mu_{2}}+\eta_{\mu_{2} \nu} p_{2 \mu_{1}}\right) p_{1 \rho(s)}\right. \\
& +\left(-\eta_{\mu_{1} \rho_{1}} p_{2 \mu_{2}} p_{1 \nu}-\eta_{\mu_{2} \rho_{1}} p_{2 \mu_{1}} p_{1 \nu}+2 \eta_{\nu \rho_{1}} p_{2 \mu_{1}} p_{2 \mu_{2}}\right) p_{1 \rho_{2}} \ldots p_{1 \rho_{s}} \\
& -\left(p_{1} \cdot p_{2}\right)\left[\left(\eta_{\mu_{1} \nu} \eta_{\mu_{2} \rho_{1}}+\eta_{\mu_{1} \rho_{1}} \eta_{\mu_{2} \nu}\right) p_{1 \rho_{2}} \ldots p_{1 \rho_{s}}\right. \\
& \quad+\left(\left(\eta_{\mu_{1} \rho_{1}} \eta_{\nu \rho_{2}}+\eta_{\mu_{1} \rho_{2}} \eta_{\nu \rho_{1}}\right) p_{2 \mu_{2}}-\left(\eta_{\mu_{1} \rho_{1}} \eta_{\mu_{2} \rho_{2}}+\eta_{\mu_{2} \rho_{1}} \eta_{\mu_{1} \rho_{2}}\right) p_{1 \nu}\right. \\
& \left.\left.\quad+\left(\eta_{\mu_{2} \rho_{1}} \eta_{\nu \rho_{2}}+\eta_{\mu_{2} \rho_{2}} \eta_{\nu \rho_{1}}\right) p_{2 \mu_{1}}\right) p_{1 \rho_{3}} \ldots p_{1 \rho_{s}}\right] \\
& \left.+\frac{1}{3}\left(p_{1} \cdot p_{2}\right)^{2}\left(\eta_{\mu_{1} \rho_{1}} \eta_{\mu_{2} \rho_{2}} \eta_{\nu \rho_{3}}+\operatorname{sym} \rho_{1,2,3}\right) p_{1 \rho_{3}} \ldots p_{1 \rho_{s}}\right\} .
\end{aligned}
$$

\section{Scattering in CHS theory: 4-vector amplitude}

We can now use the interaction terms in the CHS action found in the previous section to compute some tree-level scattering amplitudes. As the scalar $h_{0}$ is non-propagating, i.e. has zero on-shell value we will not discuss analogs of scattering amplitudes with $h_{0}$ on external legs.

The vector $h_{1}$ has the standard Maxwell kinetic term, so the definition of the corresponding 4-vector scattering amplitude is standard (the same as in the case of the external scalar scattering in [16]): we consider physical helicity \pm 1 photon states on external lines and include all exchanges with two 1-1-s vertices (2.8) connected by TT propagator for even-spin $s$ CHS field. The contribution of the $h_{0}$ exchange due to 011 vertex (2.11) exactly cancels against the contact 4 -vector vertex (2.13) as follows from (1.7) so it remains to consider only the exchanges with $s=2,4,6, \ldots$ fields on internal lines.

Before proceeding with spin 1 scattering let us note for the future discussion in sections 4 and 5 that as the CHS fields with $s>1$ in (1.2) have higher-derivative kinetic terms, the notion of S-matrix for $s>1$ external lines requires special definition. Given the free spin $s>1$ CHS equation in TT gauge $\square^{s} h_{s}=0$ one can always choose a special solution $h_{s}=h_{s}^{(0)}$ satisfying $\square h_{s}^{(0)}=0$. This equation has further on-shell gauge invariance allowing one to reduce the number of independent solutions to just 2 of a standard 2-derivative massless particle. In what follows we shall always consider only these special "physical" helicity $\pm s$ modes as the asymptotic states in the definition of the CHS S-matrix. ${ }^{14}$ Thus

\footnotetext{
${ }^{14}$ Ideally, one would like to start with a formulation of the CHS theory in terms of the set of fields with ordinary (2-derivative) kinetic terms that exists at the quadratic level [19, 20]. Unfortunately, an existence of such local action at the interacting level is an open question for $s>2$.
} 
the asymptotic states will always be massless on-shell particles with $p^{2}=0$ while the internal spin $s^{\prime}$ propagator will be $\frac{1}{p^{2 s^{\prime}}}$ times the TT projector. ${ }^{15}$

\subsection{4 -vector exchange amplitude}

Let us start with the 4-vector scattering amplitude and first set up the notation we will use. We shall consider the scattering process
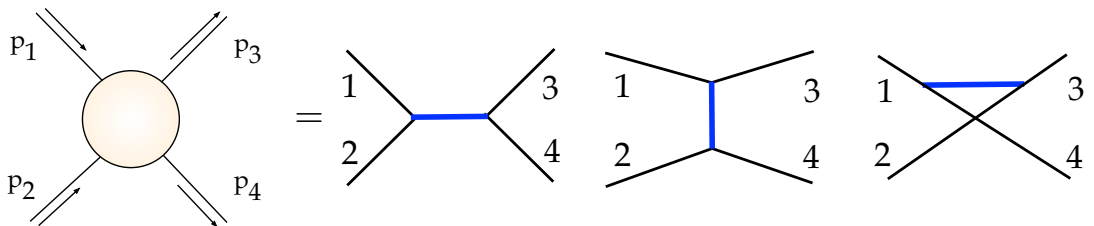

with $\left(\lambda_{1}, p_{1}\right),\left(\lambda_{2}, p_{2}\right) \rightarrow\left(\lambda_{3}, p_{3}\right),\left(\lambda_{4}, p_{4}\right)$, i.e. assume two momenta and helicities as incoming and two as outcoming with $\lambda_{i}= \pm 1$ and $p_{i}^{2}=0$. In the c.o.m. frame, we have for the momenta and polarisation vectors ${ }^{16}$

$$
\begin{aligned}
p_{1} & =(\omega, 0,0, \omega), & \varepsilon_{1}\left(p_{1}\right) & =-\frac{1}{\sqrt{2}} \lambda_{1}\left(0,1, i \lambda_{1}, 0\right) \\
p_{2} & =(\omega, 0,0,-\omega), & \varepsilon_{2}\left(p_{2}\right) & =-\frac{1}{\sqrt{2}} \lambda_{2}\left(0,-1, i \lambda_{2}, 0\right) \\
p_{3} & =(\omega, \omega \sin \theta, 0, \omega \cos \theta), & {\left[\varepsilon_{3}\left(p_{3}\right)\right]^{*} } & =-\frac{1}{\sqrt{2}} \lambda_{3}\left(0, \cos \theta,-i \lambda_{3},-\sin \theta\right) \\
p_{4} & =(\omega,-\omega \sin \theta, 0,-\omega \cos \theta), & {\left[\varepsilon_{4}\left(p_{4}\right)\right]^{*} } & =-\frac{1}{\sqrt{2}} \lambda_{4}\left(0,-\cos \theta,-i \lambda_{4}, \sin \theta\right)
\end{aligned}
$$

and the Mandelstam variables are

$$
\begin{array}{rlrl}
\mathbf{s} & =-\left(p_{1}+p_{2}\right)^{2}=4 \omega^{2}, & \mathbf{t}=-\left(p_{1}-p_{3}\right)^{2} & =-2 \omega^{2}(1-\cos \theta), \\
\mathbf{u} & =-\left(p_{1}-p_{4}\right)^{2}=-2 \omega^{2}(1+\cos \theta), & \mathbf{s}+\mathbf{t}+\mathbf{u}=0 .
\end{array}
$$

The exchange diagrams involve two 11 s vertices corresponding to $h_{1}(p) h_{1}(q) h_{s}(-p-q)$ from $(2.8)^{17}$

$$
\begin{aligned}
& \mathrm{V}_{\alpha, \beta, \rho(s)}(p, q)=\frac{1}{(s+2) !}\left\{\eta_{\alpha \beta}\left[\frac{1}{2}(p)_{\rho(s)}+\frac{1}{2}(q)_{\rho(s)}\right]\right. \\
& -\frac{1}{2} \eta_{\alpha \rho_{1}} p_{\beta} p_{\rho_{2}} \ldots p_{\rho_{s}}+\frac{1}{2} \eta_{\beta \rho_{1}} q_{\alpha} p_{\rho_{2}} \ldots p_{\rho_{s}}-\frac{1}{2} \eta_{\beta \rho_{1}} q_{\alpha} q_{\rho_{2}} \ldots q_{\rho_{s}}+\frac{1}{2} \eta_{\alpha \rho_{1}} p_{\beta} q_{\rho_{2}} \ldots q_{\rho_{s}} \\
& \left.-\frac{1}{2} \eta_{\alpha \rho_{1}} \eta_{\beta \rho_{2}} p_{\rho_{3}} \ldots p_{\rho_{s}} p \cdot q-\frac{1}{2} \eta_{\alpha \rho_{1}} \eta_{\beta \rho_{2}} q_{\rho_{3}} \ldots q_{\rho_{s}} p \cdot q\right\} .
\end{aligned}
$$

Here $h_{s}$ is assumed to be in TT gauge with the corresponding propagator (cf. (2.8))

$$
D_{\beta(s)}^{\alpha(s)}(p)=\frac{2^{s-1}(2 s+1) !}{\left(p^{2}\right)^{s}} \Pi_{\beta_{1} \cdots \beta_{s}}^{\alpha_{1} \cdots \alpha_{s}}(p)
$$

\footnotetext{
${ }^{15}$ The condition $p^{2}=0$ for the external lines will help to simplify the expressions for the required cubic CHS vertices.

${ }^{16}$ The helicity \pm 1 polarisation vector for an initial state with $p=(\omega, \omega \sin \theta, 0, \omega \cos \theta)$ is $\varepsilon_{\mu}^{( \pm)}(p)=$ $\mp \frac{1}{\sqrt{2}}(0, \cos \theta, \pm i,-\sin \theta)$. If the state is final, the polarisation vector is $\left(\varepsilon_{\mu}^{( \pm)}(p)\right)^{*}$ (see, e.g., [21, 22]).

${ }^{17}$ Here we use that $p^{2}=q^{2}=0$ for the external vector lines; $p=p_{1}, q=p_{2}$ in s-channel, etc.
} 
where the TT projector $\Pi_{\beta(s)}^{\alpha(s)}$ is built out of products of $\Pi_{\beta}^{\alpha}=\delta_{\beta}^{\alpha}-\frac{p^{\alpha} p_{\beta}}{p^{2}}$, e.g., ${ }^{18}$

$$
\begin{aligned}
\Pi_{\beta_{1} \beta_{2}}^{\alpha_{1} \alpha_{2}}= & \Pi_{\left(\beta_{1}\right.}^{\alpha_{1}} \Pi_{\left.\beta_{2}\right)}^{\alpha_{2}}-\frac{1}{3} \Pi^{\alpha_{1} \alpha_{2}} \Pi_{\beta_{1} \beta_{2}} \\
\Pi_{\beta_{1} \beta_{2} \beta_{3} \beta_{4}}^{\alpha_{1} \alpha_{2} \alpha_{3} \alpha_{4}}= & \Pi_{\left(\beta_{1}\right.}^{\left(\alpha_{1}\right.} P_{\beta_{2}}^{\alpha_{2}} P_{\beta_{3}}^{\alpha_{3}} \Pi_{\left.\beta_{4}\right)}^{\left.\alpha_{4}\right)}-\frac{6}{7} P^{\left(\alpha_{1} \alpha_{2}\right.} \Pi_{\left(\beta_{1} \beta_{2}\right.} \Pi_{\beta_{3}}^{\alpha_{3}} \Pi_{\left.\beta_{4}\right)}^{\left.\alpha_{4}\right)}+\frac{3}{35} \Pi^{\left(\alpha_{1} \alpha_{2}\right.} \Pi^{\left.\alpha_{3} \alpha_{4}\right)} \Pi_{\left(\beta_{1} \beta_{2}\right.} \Pi_{\left.\beta_{3} \beta_{4}\right)} \\
& \text { etc. }
\end{aligned}
$$

The resulting s, t, u-channel amplitudes are are given by ${ }^{19}$

$$
\begin{aligned}
& A_{\mathbf{s}}^{(s)}=2 \mathrm{~V}\left(p_{1}, p_{2}\right) \cdot D\left(p_{1}+p_{2}\right) \cdot 2 \mathrm{~V}\left(p_{3}, p_{4}\right) \cdot \varepsilon_{1} \varepsilon_{2} \varepsilon_{3}^{*} \varepsilon_{4}^{*}, \\
& A_{\mathbf{t}}^{(s)}=2 \mathrm{~V}\left(p_{1}, p_{3}\right) \cdot D\left(p_{1}-p_{3}\right) \cdot 2 \mathrm{~V}\left(p_{2}, p_{4}\right) \cdot \varepsilon_{1} \varepsilon_{2} \varepsilon_{3}^{*} \varepsilon_{4}^{*}, \\
& A_{\mathbf{u}}^{(s)}=2 \mathrm{~V}\left(p_{1}, p_{4}\right) \cdot D\left(p_{1}-p_{4}\right) \cdot 2 \mathrm{~V}\left(p_{2}, p_{3}\right) \cdot \varepsilon_{1} \varepsilon_{2} \varepsilon_{3}^{*} \varepsilon_{4}^{*} .
\end{aligned}
$$

Evaluating these amplitudes for various helicity choices we find that all amplitudes where helicity is not conserved vanish ${ }^{20}$ and for the helicity-conserving cases $\pm \pm \rightarrow \pm \pm$ or its crossing-related $\pm \mp \rightarrow \pm \mp$ we get $^{21}$

$$
\begin{aligned}
& \pm \pm \rightarrow \pm \pm: \quad A_{\mathbf{s}}^{(s)}=0, \\
& A_{\mathbf{t}}^{(s)}=c_{s}\left(\frac{\mathbf{s}}{\mathbf{t}}\right)^{s} P_{s}\left(\frac{\mathbf{t}}{\mathbf{s}}\right), \quad A_{\mathbf{u}}^{(s)}=c_{s}\left(\frac{\mathbf{s}}{\mathbf{u}}\right)^{s} P_{s}\left(\frac{\mathbf{u}}{\mathbf{s}}\right), \\
& \pm \mp \rightarrow \pm \mp: \quad A_{\mathbf{s}}^{(s)}=c_{s}\left(\frac{\mathbf{u}}{\mathbf{s}}\right)^{s} P_{s}\left(\frac{\mathbf{s}}{\mathbf{u}}\right), \quad A_{\mathbf{t}}^{(s)}=c_{s}\left(\frac{\mathbf{u}}{\mathbf{t}}\right)^{s} P_{s}\left(\frac{\mathbf{t}}{\mathbf{u}}\right), \quad A_{\mathbf{u}}^{(s)}=0 \text {. }
\end{aligned}
$$

Like in the external scalar scattering case [16] the scale invariance of the CHS theory and the fact that $h_{1}$ has canonical dimension 1 implies that the $d=4$ amplitude depends only on ratios of the Mandelstam variables. We have isolated powers of these ratios containing the internal spin $s$ CHS propagator factor in each channel (i.e. $\mathbf{t}^{-s}$ in t-channel, etc.). The remaining momentum dependence is given by the even degree $s-2$ polynomials $P_{s}(x)$ whose normalisation is fixed by the condition $P_{2}=1$ and $P_{s>2}(-1)=1$

$$
\begin{aligned}
P_{2}(x)= & 1, \quad P_{4}(x)=28+42 x+15 x^{2}, \\
P_{6}(x)= & 495+1320 x+1260 x^{2}+504 x^{3}+70 x^{4} \\
P_{8}(x)= & 8008+30030 x+45045 x^{2}+34320 x^{3}+13860 x^{4}+2772 x^{5}+210 x^{6}, \\
P_{10}(x)= & 125970+604656 x+1225224 x^{2}+1361360 x^{3}+900900 x^{4}+360360 x^{5} \\
& +84084 x^{6}+10296 x^{7}+495 x^{8} .
\end{aligned}
$$

\footnotetext{
${ }^{18}$ In general (cf. (C.3))

$$
\Pi_{\mu(s)}^{\nu(s)}=\sum_{l=0}^{\left[\frac{s}{2}\right]} a_{s, l} M_{\mu(s-2 l)}^{\nu(s-2 l)} N_{\mu(2 l)}^{\nu(2 l)}, \quad \quad a_{s, l}=\frac{(-1)^{l} s ! \Gamma\left(s-l+\frac{1}{2}\right)}{2^{2 l}(s-2 l) ! l ! \Gamma\left(s+\frac{1}{2}\right)}
$$

where $M_{\mu(p)}^{\nu(p)}=\Pi_{\mu_{1}}^{\left(\nu_{1}\right.} \ldots \Pi_{\mu_{p}}^{\left.\nu_{p}\right)}$ and $N_{\mu(2 q)}^{\nu(2 q)}=\Pi_{\left(\mu_{1} \mu_{2}\right.} \ldots \Pi_{\left.\mu_{q-1} \mu_{q}\right)} \Pi^{\left(\nu_{1} \nu_{2}\right.} \ldots \Pi^{\left.\nu_{q-1} \nu_{q}\right)}$.

${ }^{19}$ The factors of 2 in the vertices are due to the symmetry of the external lines: for a Lagrangian term $\Phi^{n}=n ! \frac{\Phi^{n}}{n !}$, the standard Feynman rules imply the coefficient $n !$.

${ }^{20}$ In standard terminology that means that only MHV amplitudes are non-zero. The same will be true for all amplitudes discussed below.

${ }^{21}$ The vanishing of the s-channel exchange for the same helicity process may be related to the fact that helicity is conserved in the 3 -point vertices where one has only the $\pm \mp$ combination (same happens for gravitational interactions, cf. [23]).
} 
The overall numerical coefficients $c_{s}$ then are

$$
c_{2}=\frac{5}{12}, \quad c_{4}=\frac{1}{20}, \quad c_{6}=\frac{13}{840}, \quad c_{8}=\frac{17}{2520}, \quad c_{10}=\frac{7}{1980} .
$$

The expressions (3.9), (3.10) were found by direct computations for $s=2, \ldots, 10$ but admit a natural generalisation to any $s$. The polynomials $P_{s}(x)$ may be expressed in terms of Jacobi polynomials $P_{n}^{(a, b)}(x)$ as $(s=2,4,6, \ldots)$

$$
P_{s}(x)=x^{s-2} P_{s-2}^{(4,0)}\left(\frac{x+2}{x}\right)
$$

while the simplest interpolating ansatz for $c_{s}$ is

$$
c_{s}=\frac{2(2 s+1)}{(s-1) s(s+1)(s+2)} .
$$

We shall provide the general derivation of (3.11), (3.12) in appendix C.

Let us mention also some useful alternative forms of the polynomials $P_{s}$ in (3.11)

$$
\begin{aligned}
P_{s}(x) & =\sum_{j=0}^{s-2}(-1)^{j}\left(\begin{array}{c}
s-2 \\
j
\end{array}\right)\left(\begin{array}{c}
2 s-j \\
s+2
\end{array}\right)(1+x)^{s-2-j} x^{j} \\
& =\left(\begin{array}{c}
2 s \\
s+2
\end{array}\right)(1+x)^{s-2}{ }_{2} F_{1}\left(2-s, 2-s,-2 s, \frac{x}{x+1}\right) \\
& =\frac{x^{2 s+1}}{(s-2) !}\left(\frac{d}{d x}\right)^{s-2} \frac{(1+x)^{s-2}}{x^{s+3}}=\sum_{j=2}^{s} \frac{1}{(j-2) !(j+2) !} \frac{(s+j) !}{(s-j) !} x^{s-j} \\
& =\frac{1}{24}(s-1) s(s+1)(s+2) x^{s-2}{ }_{2} F_{1}\left(2-s, s+3,5 ;-\frac{1}{x}\right) .
\end{aligned}
$$

For comparison, in the case of the external massless scalar scattering (coupled to CHS fields as in (2.1), (2.2) or (2.6)) the s-channel $\varphi \varphi^{*} \rightarrow \varphi \varphi^{*}$ amplitude was given [16] in terms of the Legendre polynomial $\mathrm{P}_{s}=P_{s}^{(0,0)}$

$$
A_{\mathbf{s} \varphi \varphi^{*} \rightarrow \varphi \varphi^{*}}^{(s)}=\left(s+\frac{1}{2}\right) P_{s}^{(0,0)}(-1-2 x), \quad x=\frac{\mathbf{t}}{\mathbf{s}} .
$$

One can also consider the "mixed" scattering amplitude $\varphi \varphi^{*} \rightarrow 11$ of two external conformal scalars into two vectors due to exchange of the tower of CHS fields. ${ }^{22}$ In this case the s-channel even spin s exchange amplitude is given by (cf. (3.7))

$$
A_{\mathbf{s} \varphi \varphi^{*} \rightarrow 11}^{(s)}=\mathrm{V}_{\varphi \varphi^{*} s}\left(p_{1}, p_{2}\right) \cdot D^{(s)}\left(p_{1}+p_{2}\right) \cdot 2 \mathrm{~V}\left(p_{3}, p_{4}\right) \cdot \varepsilon_{3}^{*} \varepsilon_{4}^{*},
$$

where $\mathrm{V}_{\varphi \varphi^{*} s}$ is the vertex in (2.6) and $\mathrm{V}$ is the 11 s vertex as in (3.4), (3.7). The resulting \pm \pm amplitudes vanish while the helicity-preserving $\pm \mp$ ones may be written as (cf. (3.8))

$$
A_{\mathbf{s} \varphi \varphi^{*} \rightarrow \pm 1 \mp 1}^{(s)}=k_{s} \frac{\mathbf{t} \mathbf{u}}{\mathbf{s}^{2}} Q_{s}\left(\frac{\mathbf{t}}{\mathbf{s}}\right)
$$

\footnotetext{
${ }^{22}$ Here we are assuming that one adds the action (1.3) of one conformal scalar coupled to CHS fields to the CHS action (1.2) and then studies the S-matrix of the resulting theory.
} 
where we ignore the overall minus sign and assume that numerical coefficients are defined by normalising the order $s-2$ polynomial $Q_{s}$ as $Q_{s}(-1)=1$. Explicitly, one finds $Q_{2}=$ $1, Q_{4}=\frac{1}{3}\left(3+14 x+14 x^{2}\right), \ldots$ and $k_{1}=\frac{5}{2}, k_{4}=\frac{9}{2}, \ldots$ On the basis of $s=2, \ldots, 10$ examples can guess the general $s$ expressions as

$$
Q_{s}(x)=\frac{2}{s(s-1)} P_{s-2}^{(2,2)}(-1-2 x), \quad k_{s}=s+\frac{1}{2},
$$

where $P_{s-2}^{(2,2)}$ is again the Jacobi polynomial (cf. (3.11), (3.12), (3.14)).

\section{$3.2 \quad$ Summing over spins}

As we already mentioned above, the $s=0$ exchange contribution cancels against the one of the 1111 vertex (2.13). Thus to get the total amplitude it remains to sum over all spin $s=2,4, \ldots$ exchanges. Let us consider, e.g., the $\pm \pm \rightarrow \pm \pm$ case in (3.8) (the discussion of the $\pm \mp \rightarrow \pm \mp$ case is similar) where the sum over channels is

$$
\pm \pm \rightarrow \pm \pm: \quad A^{(s)}=c_{s}\left[\left(\frac{\mathbf{s}}{\mathbf{t}}\right)^{s} P_{s}\left(\frac{\mathbf{t}}{\mathbf{s}}\right)+\left(\frac{\mathbf{s}}{\mathbf{u}}\right)^{s} P_{s}\left(\frac{\mathbf{u}}{\mathbf{s}}\right)\right] .
$$

Since $\mathbf{u}=-\mathbf{s}-\mathbf{t}$ this may be written as a function of one variable $x \equiv \frac{\mathbf{t}}{\mathbf{s}}$ as

$$
A^{(s)}=\sigma_{s}(x)+\sigma_{s}(-1-x), \quad \sigma_{s}(x) \equiv c_{s} x^{-s} P_{s}(x) .
$$

We may compute the sum over $s$ by introducing first an extra regularisation parameter $z$ and defining

$$
\sigma(x) \equiv \lim _{z \rightarrow 1} \sigma(x ; z), \quad \sigma(x ; z) \equiv \sum_{s=2,4,6, \ldots}^{\infty} \sigma_{s}(x) z^{s-2}
$$

Let us first omit the overall coefficient $c_{s}$ in $\sigma_{s}$ and consider the formal sum over all (even and odd) $s=2,3,4, \ldots$

$$
K(x ; z) \equiv \sum_{s=2}^{\infty} x^{-s} P_{s}(x) z^{s-2} .
$$

This can be written in a closed form using the generating function for the Jacobi polynomials $P_{s-2}^{(4,0)}[24]$ as

$$
K(x ; z)=\frac{16}{x^{2}}\left[\sqrt{z^{2}-\frac{2 z(x+2)}{x}+1}\right]^{-1}\left[\sqrt{z^{2}-\frac{2 z(x+2)}{x}+1}-z+1\right]^{-4} .
$$

Then using the fact that $c_{s}$ in (3.12) admits the following representation

$$
c_{s}=\frac{1}{s+2}-\frac{1}{s+1}+\frac{1}{s-1}-\frac{1}{s},
$$

we can compute $\sigma(x ; z)$ by multiplying $(3.22)$ by a suitable power of $z$, integrating, and then dividing by another appropriate power of $z$. Finally, the sum over spins may be restricted to even $s$ only by simply taking one half of the sum of the expressions with $z$ 
and with $-z$. While the resulting expression is quite cumbersome, its $z \rightarrow 1$ limit turns out to be finite and simple

$$
\sigma(x)=x(x+1) \log \frac{x+1}{x}-x-\frac{1}{2} .
$$

As it is easy to check, this function satisfies the relation $\sigma(x)=-\sigma(-1-x)$ implying that the total summed-over-spins amplitude vanishes:

$$
A(x)=\sum_{s=2,4,6, \ldots}^{\infty} A^{(s)}(x)=\sigma(x)+\sigma(-1-x)=0 .
$$

Here we formally assumed that $\sigma(x)$ is defined for any $x$ using analytic continuation. In fact, this function is real for $x \in[-\infty,-1] \cup[0, \infty]$ while the argument of the amplitude in (3.19) is $x=\frac{\mathbf{t}}{\mathbf{s}}=-\frac{1}{2}(1-\cos \theta) \in[-1,0]$. In the latter "physical" interval one finds again that $A(x+i 0)=0$ for any sign of the infinitesimal imaginary part. In appendix $\mathrm{B}$ we provide an independent check of the vanishing of the amplitude (3.25) at the special kinematical point $\mathbf{u}=0$ or $x=-1$ (or, equivalently, at $x=0$ ).

Another clarification is that in the above discussion we have excluded the special points $x=0,-1$ where the amplitude may have delta-function singularities as in the external scalar amplitude case [16]. Indeed, as was shown in [16], the sum of the Legendre polynomials in (3.14) is given by $\sum_{s=0}^{\infty}\left(s+\frac{1}{2}\right) \mathrm{P}_{s}(x)=\delta(x-1)$, so the total amplitude given by the sum of the s- and t-channels is $\sim \delta\left(\frac{\mathbf{u}}{\mathbf{s}}\right)+\delta\left(\frac{\mathbf{u}}{\mathbf{t}}\right)$ which vanishes for real momenta. Similar cancellation happens here as well as we show in appendix B.

\section{General structure of CHS exchange amplitudes}

To generalise the above vector scattering results to higher $s>1$ spin scattering case it is useful first to discuss the structure of the CHS 4-particle amplitudes expected on the basis of Lorentz and scale invariance. It turns out that the appearance of the special Jacobi polynomials in (3.11), (3.14) and (3.17) is not accidental and may be related to the partial wave expansion of the $\lambda_{1}, \lambda_{2} \rightarrow \lambda_{3}, \lambda_{4}$ transition amplitude discussed by Jacob and Wick [17] (see also [25, 26]).

Considering the c.o.m. frame and using the completeness of states relation one can represent generic scattering amplitude as a sum over on-shell states of a massive particle with mass $=\sqrt{\mathbf{s}}$ and $\operatorname{spin} J[17]$

$$
\begin{aligned}
A_{\left\{\lambda_{i}\right\}}(\mathbf{s}, \theta) & =R_{\left\{\lambda_{i}\right\}}(\theta) \sum_{J \geq M}\left(J+\frac{1}{2}\right) \mathrm{F}_{\left\{\lambda_{i}\right\}}^{(J)}(\mathbf{s}) P_{J-M}^{(|\lambda-\mu|,|\lambda+\mu|)}(\cos \theta), \\
\lambda & =\lambda_{1}-\lambda_{2}, \quad \mu=\lambda_{3}-\lambda_{4}, \quad M=\max (|\lambda|,|\mu|), \\
R_{\left\{\lambda_{i}\right\}}(\theta) & =\left(\cos \frac{\theta}{2}\right)^{|\lambda+\mu|}\left(\sin \frac{\theta}{2}\right)^{|\lambda-\mu|}=\left(-\frac{\mathbf{u}}{\mathbf{s}}\right)^{\frac{1}{2}|\lambda+\mu|}\left(-\frac{\mathbf{t}}{\mathbf{s}}\right)^{\frac{1}{2}|\lambda-\mu|} .
\end{aligned}
$$

Here $\left\{\lambda_{i}\right\}=\left(\lambda_{1}, \lambda_{2} ; \lambda_{3}, \lambda_{4}\right), \cos \theta=1+2 \frac{\mathbf{t}}{\mathbf{s}}$ and $P_{k}^{(a, b)}$ is the Jacobi polynomial. The latter originates from the expression for the spherical $d$-function $(N=\min (|\lambda|,|\mu|))$

$$
d_{\lambda \mu}^{J}(\theta)=\sqrt{\frac{(J+M) !(J-M) !}{(J+N) !(J-N) !}} R_{\left\{\lambda_{i}\right\}}(\theta) P_{J-M}^{(|\lambda-\mu|,|\lambda+\mu|)}(\cos \theta) .
$$


We assume that the scattering particles are massless. If the theory is scale-invariant (has no dimensional parameters) the dependence of the coefficient functions $\mathrm{F}_{\left\{\lambda_{i}\right\}}^{(J)}$ on $\mathbf{s}$ should be controlled only by dimensions $\Delta_{i}$ of the scattering fields,

$$
\mathrm{F}_{\left\{\lambda_{i}\right\}}^{(J)}(\mathbf{s})=F_{\left\{\lambda_{i}\right\}}^{(J)} \mathbf{s}^{\Delta}, \quad \Delta \equiv \frac{1}{2}\left(4-\sum_{i=1}^{4} \Delta_{i}\right) .
$$

Here $F_{\left\{\lambda_{i}\right\}}^{(J)}$ are some numerical coefficients encoding dynamical information about a particular theory. For example, $\Delta=0$ for scattering of dimension 1 massless scalars or vectors in $4 \mathrm{~d}$. In the case of CHS scattering with asymptotic states chosen, as discussed in the previous section, to be the standard massless spin $\left|\lambda_{i}\right|$ particles the representation (4.1), (4.5) should again apply with $\Delta=\frac{1}{2}\left(\sum_{i}\left|\lambda_{i}\right|-4\right)$ (the CHS field dimensions are $\Delta_{i}=2-\left|\lambda_{i}\right|$ ).

Our key observation is that in the present context of conformal higher spin theory the spin $J$ contribution in (4.1) should have the same structure as the contribution of an intermediate CHS field exchange with $s=J$ in s-channel. This should be a kinematical consequence of the fact that a massive $\left(m^{2}=\mathbf{s}\right)$ intermediate spin $J$ state in (4.1) may be described by a totally symmetric field $\phi_{\mu_{1} \ldots \mu_{J}}$ satisfying $\left(-\square+m^{2}\right) \phi_{\mu_{1} \ldots \mu_{J}}=0$ as well as the tracelessness and transversality conditions (leaving only $2 J+1$ states as physical degrees of freedom). At the same time, the CHS scattering is also mediated by the TT field exchange with the propagator in (3.5). The only formal difference is in the overall s-dependence that appears in F but in the CHS scattering case the latter is controlled by the scale invariance leading to (4.5).

This formal interpretation of the spin $J$ term in (4.1) as the CHS spin $s=J$ exchange amplitude should directly apply only to the s-channel exchange: this is due to the selection of $\mathbf{s}$ variable as the c.o.m. frame mass parameter in (4.1) and thus as the variable that should appear in the propagator of the corresponding exchanged CHS field. The total CHS amplitude given by the sum over all channels as in (3.1), (3.18) will also have the general form (4.1) when expanded in the Jacobi polynomials but the $J=s$ identification of the particular term in the sum with the contribution of the CHS exchange will be valid only in a particular channel (in s-channel or after renaming the kinematic variables and helicities also in t- and u-channels, see below).

Another remark is that this identification of the $J$-term in (4.1) with the higher spin exchange does not apply to the case of the 2-derivative massless higher spin scattering in flat space discussed in [27]. The reason is that the massless spin $s$ propagator (taken, e.g., in the de Donder gauge) is not traceless-transverse and thus the massless higher spin particle exchange can not be directly identified with a massive spin $J$ on-shell state contribution in the sum in (4.1)..$^{23}$

Let us now see how the previously discussed cases of the external scalar and vector scattering (3.8), (3.14), (3.16) via the CHS exchange may be related to (4.1). In the case

\footnotetext{
${ }^{23}$ Indeed, the scattering amplitude for four massless scalars exchanging the tower of massless higher spins was given in [27] by the sum of the Chebyshev polynomials rather than the Legendre polynomials appearing (3.14) in the case of the conformal higher spin exchange in [16]. Interestingly, there is still a formal relation between 4 -scalar scattering via massless higher spin exchange in $d+1$ dimensions and via conformal higher spin exchange in $d$ dimensions suggesting possible AdS/CFT connection.
} 
of the $\varphi \varphi^{*} \rightarrow \varphi \varphi^{*}$ scattering we have $\lambda_{i}=0, \lambda=\mu=0, M=0$, and thus should expect, according to (4.1), (4.5), to find the s-channel spin $J$ contribution to be

$$
A_{\mathbf{s} 0,0 ; 0,0}^{(J)}(\mathbf{s}, \cos \theta)=\left(J+\frac{1}{2}\right) F_{0,0 ; 0,0}^{(J)} P_{J}^{(0,0)}(\cos \theta) .
$$

Comparing this with the s-channel result (3.14) of the direct computation using that $\cos \theta=$ $1+2 \frac{\mathrm{t}}{\mathrm{s}}$ we conclude that the two expression indeed match provided $s=J$ and

$$
F_{0,0 ; 0,0}^{(s)}=1 .
$$

For $\varphi \varphi^{*} \rightarrow \pm \mp$ in (3.15), (3.16) we have $\lambda=\lambda_{1}=\lambda_{2}=0, \lambda_{3}=-\lambda_{4}= \pm 1, \mu=M= \pm 2$, and thus from (4.1), (4.5) should get

$$
A_{\mathbf{s} 0,0 ; \pm 1, \mp 1}^{(J)}(\mathbf{s}, \cos \theta)=\left(J+\frac{1}{2}\right) F_{0,0 ; \pm 1, \mp 1}^{(J)} \frac{\mathbf{u} \mathbf{t}}{\mathbf{s}^{2}} P_{J-2}^{(2,2)}(\cos \theta) .
$$

Comparing this with (3.16), (3.17) we find perfect match provided $s$ is identified with $J$ (which should be taken to be even) and ${ }^{24}$

$$
F_{0,0 ; \pm \mp}^{(s)}=\frac{2}{s(s-1)} .
$$

In the case of $\pm 1 \pm 1 \rightarrow \pm 1 \pm 1$ scattering in (3.8) we have the two contributions of the tand $\mathrm{u}$ - channels that are to be analysed separately. For example, considering the t-channel exchange to be able to compare it to (4.1) we should first re-interpret it as an s-channel exchange by relabelling the states and Mandelstam variables. Explicitly, the t-channel scattering of original " $\mathrm{X}$ "-particles may be interpreted as s-channel scattering of effective "Y"-particles, i.e. $X_{1}+\bar{X}_{3} \rightarrow X_{4}+\bar{X}_{2}$ is equivalent to $Y_{1}+Y_{2} \rightarrow Y_{3}+Y_{4}$. For the Yparticles we then have $\lambda_{1}=-\lambda_{2}= \pm 1, \lambda_{3}=-\lambda_{4}= \pm 1, \lambda=\mu=2, \quad M=2$ and thus from (4.1), (4.5) we should get

$$
A_{\mathbf{s} \pm 1, \mp 1 ; \mp 1, \pm 1}^{(J)}=\left(J+\frac{1}{2}\right) F_{ \pm 1, \mp 1 ; \mp 1, \pm 1}^{(J)} \frac{\mathbf{u}_{Y}^{2}}{\mathbf{s}_{Y}^{2}} P_{J-2}^{(0,4)}\left(\cos \theta_{Y}\right), \quad \cos \theta_{Y}=-1-2 \frac{\mathbf{u}_{Y}}{\mathbf{s}_{Y}} .
$$

The Y-kinematics becomes the X-kinematics after $\mathbf{s}_{Y} \rightarrow \mathbf{t}, \mathbf{t}_{Y} \rightarrow \mathbf{u}, \mathbf{u}_{Y} \rightarrow \mathbf{s}$. Thus for the t-channel exchange of the $\mathrm{X}$-particles we should get

$$
A_{\mathbf{t} \pm 1, \pm 1 ; \pm 1, \pm 1}^{(J)}(\mathbf{t}, \cos \theta)=\left(J+\frac{1}{2}\right) F_{ \pm 1, \pm 1 ; \pm 1, \pm 1}^{(J)} \frac{\mathbf{s}^{2}}{\mathbf{t}^{2}} P_{J-2}^{(0,4)}\left(-1-2 \frac{\mathbf{s}}{\mathbf{t}}\right)
$$

This matches the t-channel result in (3.8), (3.11), (3.12) with $J=s$ since

$$
\left(\frac{\mathbf{s}}{\mathbf{t}}\right)^{s}\left(\frac{\mathbf{t}}{\mathbf{s}}\right)^{s-2} P_{s-2}^{(4,0)}\left(1+2 \frac{\mathbf{s}}{\mathbf{t}}\right)=\frac{\mathbf{s}^{2}}{\mathbf{t}^{2}} P_{s-2}^{(0,4)}\left(-1-2 \frac{\mathbf{s}}{\mathbf{t}}\right)
$$

\footnotetext{
${ }^{24}$ Note that the restriction that $J=s$ should be even does not follow from (4.1) and is an extra dynamical property of CHS theory (parity invariance of the original scalar theory (2.1) implying the absence 1-1-s vertices with odd $s$ ). For even $s$ the polynomial $P_{s-2}^{(2,2)}(x)$ is even.
} 
provided also we choose

$$
F_{ \pm 1, \pm 1 ; \pm 1, \pm 1}^{(s)}=\frac{c_{s}}{s+\frac{1}{2}}=\frac{4}{(s-1) s(s+1)(s+2)}
$$

Guided by the above three examples (4.7), (4.9) and (4.13) we may conjecture the general dependence of $F_{\left\{\lambda_{i}\right\}}^{(J)}$ in (4.5) on $J=s$ in the case of CHS exchange amplitudes to be ${ }^{25}$

$$
F_{\left\{\lambda_{i}\right\}}^{(s)}=\mathrm{k}_{\lambda, \mu} \frac{(s-M) !}{(s+N) !}, \quad N=\min (|\lambda|,|\mu|), \quad M=\max (|\lambda|,|\mu|) .
$$

Then for $\mathrm{k}_{\lambda, \mu}=1$ we indeed get $F_{\{0\}}^{(s)}$ as in (3.11), $F_{0,0 ; \pm \mp}^{(s)}$ as in (4.9) and $F_{\{ \pm 1\}}^{(s)}$ as in (4.13) (where one should, as explained above, use $\lambda=\mu=2$ to match the t-channel result). It turns out that this ansatz (4.14) applies also in all other cases discussed below.

\section{Scattering amplitudes with conformal gravitons}

Let us now turn to the discussion of conformal graviton scattering due to the exchange of the CHS fields. The relevant 2-2-s interaction vertex was given in (2.15). As discussed in section 3, we shall be scattering only the "physical" massless spin 2 component of the conformal spin 2 field, attaching the corresponding asymptotic states to the amputated Green's functions. ${ }^{26}$

\section{$5.1 \quad 22 \rightarrow 22$ scattering}

Let us first discuss what we should expect to get for the structure of the $22 \rightarrow 22$ even spin $s \geq 4$ exchange on general symmetry grounds. We shall assume that as in the 4 -vector case

\footnotetext{
${ }^{25}$ One reason why this choice may be special is the following property of the Jacobi polynomials: $(\lambda+\mu) ! \frac{(s-M) !}{(s+N) !} P_{s-M}^{(|\lambda-\mu|, \lambda+\mu)}(-1)=1$ where $\lambda$ and $\mu$ should be integer and $s-M$ should be even integer. This implies the $s$-independence of the backward scattering amplitude in the direct channel.

${ }^{26}$ Let us note also that using the standard formulation of the CHS action (1.2) one may also study the scattering of other "ghost"-like modes described by the higher-derivative CHS equations. In general, the 6 dynamical degrees of freedom of the Weyl graviton way be described by the collection of the standard massless spin 2 field, massless vector, and massless spin 2 ghost field states (for a discussion of solutions of linearised Weyl gravity equations reproducing the dynamical degrees of freedom count [28-30] see [31] and also appendix $\mathrm{C}$ in [19]). Explicitly, choosing the TT gauge $\left(h_{m}^{m}=0, \partial^{m} h_{m n}=0\right)$ we get the free conformal graviton equation as $\square^{2} h_{m n}=0$ which is solved by $h_{m n}=h_{m n}^{(1)}+h_{m n}^{(2)}=\left(a_{m n}+b_{m n} u_{k} x^{k}\right) e^{i p \cdot x}+c . c$. where $p^{2}=0, u^{2}=-1, u \cdot p \neq 0, \quad a_{m}^{m}=b_{m}^{m}=0$. Here $h_{m n}^{(1)}$ represents the massless spin 2 and spin 1 modes and $h_{m n}^{(2)}$ the ghost-like spin 2 mode (which grows in time and leads to negative energy contributions). Using the Lorentz symmetry and the residual gauge freedom one may choose [31]: $p^{m}=(p, 0,0, p), u^{m}=(1,0,0,0)$, $a_{11}+a_{22}=b_{11}+b_{22}=0, \quad a_{m 3}=b_{m 3}=b_{m 0}=0$ and then the $2+2+2$ dynamical d.o.f. are described by the helicity \pm 2 tensor $\left(a_{11} \pm i a_{12}\right) e^{i p \cdot x}$, helicity \pm 1 vector $\left(a_{01} \pm i a_{02}\right) e^{i p \cdot x}$ and helicity \pm 2 ghost tensor $\left(b_{11} \pm i b_{12}\right) x^{0} e^{i p \cdot x}$. The spin 1 and ghost spin 2 become parts of massive spin 2 ghost if one adds the $R$ term to Weyl action to get a diagonal mode decomposition. At the level of the flat-space partition function
} of Weyl graviton the above $2+2+2$ split corresponds to the following decomposition [1]:

$$
Z_{2}=\left[\frac{\left(\operatorname{det} \Delta_{1}\right)^{3}}{\left(\operatorname{det} \Delta_{2}\right)^{2}}\right]^{1 / 2}=\left[\frac{\operatorname{det} \Delta_{1} \perp}{\operatorname{det} \Delta_{2} \perp}\right]^{1 / 2}\left[\frac{\operatorname{det} \Delta_{0} \perp}{\operatorname{det} \Delta_{2} \perp}\right]^{1 / 2}=\left[\frac{\operatorname{det} \Delta_{1 \perp}}{\operatorname{det} \Delta_{2} \perp}\right]^{1 / 2}\left[\frac{\operatorname{det} \Delta_{0} \perp}{\operatorname{det} \Delta_{1} \perp}\right]^{1 / 2}\left[\frac{\operatorname{det} \Delta_{1} \perp}{\operatorname{det} \Delta_{2} \perp}\right]^{1 / 2}
$$

Here $\Delta_{s}$ are 2-derivative Laplacians defined on traceless rank $s$ symmetric fields. 
the non-vanishing scattering amplitudes should be similar to (3.8) (where now \pm will stand for \pm 2 helicities of the external massless graviton state). Thus for the $++\rightarrow++$ amplitude we should have the contributions from the $\mathrm{t}$ - and $\mathrm{u}$ - channels. ${ }^{27}$ Then repeating the analysis that in the vector case lead to (4.11) we conclude that for the t-channel exchange of CHS spin $J$ states we should expect from (4.1), (4.5) to find for $J \geq 4$

$$
A_{\mathbf{t} \pm 2, \pm 2 ; \pm 2, \pm 2}^{(J)}(\mathbf{t}, \cos \theta)=\left(J+\frac{1}{2}\right) F_{ \pm 2, \pm 2 ; \pm 2, \pm 2}^{(J)} \mathbf{t}^{2} \frac{\mathbf{s}^{4}}{\mathbf{t}^{4}} P_{J-4}^{(0,8)}\left(-1-2 \frac{\mathbf{s}}{\mathbf{t}}\right)
$$

Here $\mathbf{t}^{2}$ factor reflects the fact that the conformal graviton has dimension 0 (cf. (4.5)). The total amplitude due to spin $s=J$ exchange should then be as in (3.18) (cf. (3.11))

$$
\begin{aligned}
\pm 2 \pm 2 \rightarrow \pm 2 \pm 2: \quad A^{(s)} & =c_{s} \mathbf{s}^{2}\left[\left(\frac{\mathbf{s}}{\mathbf{t}}\right)^{s-2} P_{s}\left(\frac{\mathbf{t}}{\mathbf{s}}\right)+\left(\frac{\mathbf{s}}{\mathbf{u}}\right)^{s-2} P_{s}\left(\frac{\mathbf{u}}{\mathbf{s}}\right)\right] \\
P_{s}(x) & =x^{s-2} P_{s-4}^{(8,0)}\left(\frac{x+2}{x}\right) .
\end{aligned}
$$

If we also assume the validity of the conjecture (4.14) for the coefficients $F_{\left\{\lambda_{i}\right\}}^{(J)}$ then we may expect also to get

$$
c_{s}=\mathrm{k} \frac{2 s+1}{(s-3)(s-2)(s-1) s(s+1)(s+2)(s+3)(s+4)},
$$

where $\mathrm{k}$ is some $s$-independent numerical factor.

Remarkably, the direct computation based on the CHS action and carried out for several even ${ }^{28}$ values of $s \geq 4$ confirms the above expressions (5.2), (5.4) and fixes $\mathrm{k}$ in (5.4) to be

$$
\mathrm{k}=\frac{9}{8}
$$

Similar result is found for the $\pm 2 \mp 2 \rightarrow \pm 2 \mp 2$ exchange (cf. (3.8)). The general derivation of (5.2)-(5.5) may be given using the same formalism as described for the $11 \rightarrow 11$ case in appendix C.

We can now sum the amplitude (5.2) over all even $s=4,6, \ldots$ using the same method as in the vector scattering case (3.19)-(3.24):

$$
\begin{aligned}
\sum_{s=4,6, \ldots}^{\infty} A^{(s)}(x) & =\mathbf{s}^{2}[\sigma(x)+\sigma(-1-x)], \quad x \equiv \frac{\mathbf{t}}{\mathbf{s}} \\
\sigma(x) & =\lim _{z \rightarrow 1} \sum_{s=4,6, \ldots}^{\infty} c_{s} x^{2-s} P_{s}(x) z^{s-4} .
\end{aligned}
$$

\footnotetext{
${ }^{27}$ We again assume two incoming and two outgoing momenta; choosing all momenta as incoming this becomes the MHV ++-- amplitude.

${ }^{28}$ Recall that the $2-2 s$ vertex (2.15) vanishes for odd $s$.
} 
After a rather involved computation using the generating function for the Jacobi polynomials in (5.3) we found that ${ }^{29}$

$$
\sigma(x)=\frac{1}{4320}\left[60(x+1)^{3} x^{3} \log \frac{x+1}{x}-60 x^{5}-150 x^{4}-110 x^{3}-15 x^{2}+3 x-1\right] .
$$

One can then check that the combination of the $\sigma$ functions appearing in (5.6) vanishes as in the vector exchange case (3.25)

$$
\sigma(x)+\sigma(-1-x)=0,
$$

i.e. the t- and $\mathrm{u}$-channel contributions summed over $s=4,6, \ldots$ cancel against each other.

To find the total $22 \rightarrow 22$ amplitude one is still to add (i) the contributions of the lowspin $s<4$ CHS exchanges (i.e. the exchange mediated by the non-propagating spin 0 field $h_{0}$ and the exchange of the spin 2 conformal graviton itself) and also (ii) the contribution of the 2222 contact vertex that is found from the UV singular part of the diagram (2.12) with four spin 2 current insertions (with vertices in (2.6) as the external legs are assumed to be TT). We found the following expressions for the spin 0 exchanges with the cubic vertex in (2.16):

$$
\begin{aligned}
& \pm 2 \pm 2 \rightarrow \pm 2 \pm 2: \quad A_{\mathbf{s}}^{(0)}=\frac{\mathbf{s}^{2}}{4608}, \quad A_{\mathbf{t}}^{(0)}=\frac{\mathbf{t}^{2} \mathbf{u}^{4}}{512 \mathbf{s}^{4}}, \quad A_{\mathbf{u}}^{(0)}=\frac{\mathbf{t}^{4} \mathbf{u}^{2}}{512 \mathbf{s}^{4}}, \\
& \pm 2 \mp 2 \rightarrow \pm 2 \mp 2: \quad A_{\mathbf{s}}^{(0)}=0, \quad A_{\mathbf{t}}^{(0)}=\frac{\mathbf{t}^{2} \mathbf{u}^{4}}{512 \mathbf{s}^{4}}, \quad A_{\mathbf{u}}^{(0)}=\frac{(\mathbf{s}+3 \mathbf{t})^{2} \mathbf{u}^{4}}{4608 \mathbf{s}^{4}} \text {. }
\end{aligned}
$$

The spin 2 exchanges (with the 2-2-2 vertices as in (2.15)) are

$$
\begin{array}{ll} 
\pm 2 \pm 2 \rightarrow \pm 2 \pm 2: & A_{\mathbf{s}}^{(2)}=\frac{\mathbf{s}^{2}+6 \mathbf{s t}+6 \mathbf{t}^{2}}{23040} \\
A_{\mathbf{t}}^{(2)} & =\frac{\mathbf{u}^{2}\left(2 \mathbf{s}^{4}-10 \mathbf{s}^{3} \mathbf{t}+33 \mathbf{s}^{2} \mathbf{t}^{2}-24 \mathbf{s} \mathbf{t}^{3}+3 \mathbf{t}^{4}\right)}{7680 \mathbf{s}^{4}} \\
A_{\mathbf{u}}^{(2)} & =\frac{\mathbf{t}^{2}\left(2 \mathbf{s}^{4}-10 \mathbf{s}^{3} \mathbf{u}+33 \mathbf{s}^{2} \mathbf{u}^{2}-24 \mathbf{s} \mathbf{u}^{3}+3 \mathbf{u}^{4}\right)}{7680 \mathbf{s}^{4}} \\
\pm 2 \mp 2 \rightarrow \pm 2 \mp 2: & A_{\mathbf{s}}^{(2)}=0, \quad A_{\mathbf{t}}^{(2)}=\frac{\mathbf{u}^{4}\left(2 \mathbf{s}^{2}+2 \mathbf{s} \mathbf{t}+3 \mathbf{t}^{2}\right)}{7680 \mathbf{s}^{4}} \\
A_{\mathbf{u}}^{(2)} & =\frac{\mathbf{u}^{4}\left(10 \mathbf{s}^{2}+18 \mathbf{s} \mathbf{u}+9 \mathbf{u}^{2}\right)}{23040 \mathbf{s}^{4}} .
\end{array}
$$

The contributions of the 4 -derivative 2222 contact vertex which is the $s=2$ analog of (2.13) are found to be

$$
\begin{aligned}
& \pm 2 \pm 2 \rightarrow \pm 2 \pm 2: \quad A^{\text {(cont) }}=-\frac{\mathbf{s}^{6}-\mathbf{s}^{5} \mathbf{t}+26 \mathbf{s}^{4} \mathbf{t}^{2}+63 \mathbf{s}^{3} \mathbf{t}^{3}+54 \mathbf{s}^{2} \mathbf{t}^{4}+27 \mathbf{s} \mathbf{t}^{5}+9 \mathbf{t}^{6}}{1920 \mathbf{s}^{4}} \\
& \pm 2 \mp 2 \rightarrow \pm 2 \mp 2: \quad A^{\text {(cont) }}=-\frac{\mathbf{u}^{4}\left(\mathbf{s}^{2}+3 \mathbf{s t}+9 \mathbf{t}^{2}\right)}{1920 \mathbf{s}^{4}} .
\end{aligned}
$$

\footnotetext{
${ }^{29}$ Let us note a similarity in the structure of (5.6) and (3.24). This suggests that for higher spin jj $\rightarrow \mathrm{jj}$ scattering one may be able to guess the expression for $\sigma(x)$ and then check that the coefficients in its expansion in a suitable set of Jacobi polynomials reproduces the $c_{s}$ prefactor. Similar ideas have been exploited in [32].
} 
Remarkably, the sum of these three contributions vanishes for each of the helicity choices:

$$
\left[A_{\mathbf{s}}^{(0)}+A_{\mathbf{t}}^{(0)}+A_{\mathbf{u}}^{(0)}\right]+\left[A_{\mathbf{s}}^{(2)}+A_{\mathbf{t}}^{(2)}\right]+A_{\mathbf{u}}^{(2)}+A^{(\text {cont })}=0 .
$$

Note that this result is equivalent to the vanishing of the 4-graviton scattering amplitude in the non-linear $C^{2}$ Weyl gravity theory. Indeed, the linear scalar - CHS coupling action (1.3) is equivalent to the covariant conformal scalar action (1.4) by a local field redefinition (1.6). As the latter action directly leads to the Weyl tensor squared action as the "induced" one (1.5), and as the field redefinitions of $h_{0}$ and $h_{2}$ in the spin $\leq 2$ part of the CHS action induced from (1.3), (2.1) should not change the graviton S-matrix, the latter should be same as in the Weyl theory. In more detail, adding the exchange of the non-propagating $h_{0}$ field produces (as it follows from (2.16)) an extra 4-derivative 2222 contact vertex contribution. The remaining local redefinition of $h_{\mu \nu}$ in (1.6) may alter the 222 vertices by terms proportional to the linearised equations of motion $\left(\square^{2} h_{\mu \nu}^{\mathrm{TT}}=0\right)$ and also change the quartic 2222 vertex, but it cannot change the resulting on-shell 4-graviton scattering amplitude.

The vanishing of the tree-level 4-graviton amplitude in Weyl theory can be deduced also from the expressions in [33] for the massless graviton scattering in $L=a R+b C^{2}$ theory by taking the limit $a \rightarrow 0$ in the final expression for the 4 -graviton amplitude. The propagator here is symbolically $\frac{1}{a p^{2}+b p^{4}}[34]$ (reducing to the Weyl graviton propagator for $a \rightarrow 0$ or to the Einstein propagator for $b \rightarrow 0)$ so as long as the asymptotic states are chosen to be massless helicity \pm 2 gravitons the resulting amplitude interpolates smoothly between the standard Einstein 4 -graviton one and zero in the Weyl theory. ${ }^{30}$

Remarkably, as we have just seen, the vanishing of tree-level 4-graviton amplitude in Weyl theory generalises to the full CHS theory: the results (5.6), (5.9) and (5.13) combined together imply that like the $11 \rightarrow 11$ amplitude in (3.25) (and also the external conformal scalar amplitude [16]) the total $22 \rightarrow 22$ conformal graviton scattering amplitude in the CHS theory vanishes after all intermediate exchange contributions are added together.

\section{$5.2 \quad 11 \rightarrow 22$ scattering}

One may also consider some "mixed" 4-particle amplitudes involving both vectors and conformal gravitons. The amplitudes with odd number of vectors vanish identically so one is to consider only $11 \rightarrow 22$ case. Here the two a priori non-trivial helicity choices are $\pm 1 \mp 1 \rightarrow \pm 2 \mp 2$ and $\pm 1 \pm 1 \rightarrow \pm 2 \mp 2$.

Let us first briefly mention also the expressions for the "mixed" amplitude where two external conformal scalars $\varphi$ in (2.1) scatter into two conformal gravitons. As in

\footnotetext{
${ }^{30}$ Let us also mention that the conformal graviton amplitudes in flat space were computed in [35] starting with the twistor string theory of [36]. The latter should be related to "non-minimal" conformal supergravity containing extra dimension 0 scalar coupling to Weyl squared term, $\phi \square^{2} \phi+(1+k \phi+\ldots) C^{2}+\ldots$. The tree-level 4-graviton amplitude in such theory is given by the sum of the 4-graviton amplitude in Weyl theory and the scalar exchange $\sim k^{2} C^{2} \square^{-2} C^{2}$. The non-zero result for the 4-graviton amplitude found in [35] appears to be given just by this scalar exchange, i.e. is consistent with the vanishing of the graviton amplitude in pure Weyl theory. Similar result was found in $[37,38]$ by taking the flat limit of the conformal graviton scattering amplitude in $\mathrm{dS}$ space which is the same as the Einstein gravity one [39] times the cosmological constant factor.
} 
the $\varphi \varphi^{*} \rightarrow 11$ case (3.15) the non-vanishing helicity-preserving amplitude $\varphi \varphi^{*} \rightarrow \pm 2 \mp 2$ receives contributions from even spin $s \geq 4$ exchanges that should have the general structure consistent again with $J=s$ term in (4.1), (4.5):

$$
A_{\mathbf{s} 0,0 ; \pm 2, \mp 2}^{(s)}=\left(s+\frac{1}{2}\right) F_{0,0 ; \pm 2, \mp 2}^{(s)} \mathbf{s}\left(\frac{\mathbf{u} \mathbf{t}}{\mathbf{s}^{2}}\right)^{2} P_{s-4}^{(4,4)}\left(-1-2 \frac{\mathbf{t}}{\mathbf{s}}\right) .
$$

The explicit computation for $c_{s}=\left(s+\frac{1}{2}\right) F_{0,0 ; \pm 2, \mp 2}^{(s)}$ gives again the result consistent with the ansatz (4.14) (here $\lambda=0, \mu=4, M=4, N=0$ )

$$
c_{s}=\left(s+\frac{1}{2}\right) F_{0,0 ; \pm 2, \mp 2}^{(s)}=-\frac{3}{4} \frac{2 s+1}{(s-3)(s-2)(s-1) s} .
$$

To get the full amplitude one is to add also the contributions of the $s=0,2$ exchanges.

Turning to the $\pm 1 \mp 1 \rightarrow \pm 2 \mp 2$ amplitude, we find that the non-vanishing helicitypreserving even $s \geq 4$ exchange amplitude in the s-channel has again the form as predicted by (4.1), (4.5)

$$
\begin{aligned}
A_{\mathbf{s}}^{(s)} \pm 1, \mp 1 ; \pm 2, \mp 2 & =c_{s} \mathbf{s} \frac{\mathbf{t} \mathbf{u}^{3}}{\mathbf{s}^{4}} P_{s-4}^{(6,2)}\left(-1-2 \frac{\mathbf{t}}{\mathbf{s}}\right) \\
c_{s} & =\left(s+\frac{1}{2}\right) F_{ \pm 1, \pm 1 ; \pm 2, \mp 2}^{(s)}=\frac{3}{2} \frac{2 s+1}{(s-3)(s-2)(s-1) s(s+1)(s+2)},
\end{aligned}
$$

where $\lambda=2, \mu=4, M=4, N=2$ so the expression for $c_{s}$ is again consistent with (4.14). In the t-channel one finds (after an appropriate relabelling of helicities and kinematic variables) that for odd $s \geq 3^{31}$

$$
\begin{aligned}
A_{\mathbf{s}}^{(s)}{ }_{1, \mp 1 ; \pm 2, \mp 2} & =c_{s}^{\prime} \mathbf{s} \frac{\mathbf{u}^{3}}{\mathbf{s t}^{2}} P_{s-4}^{(6,0)}\left(-1-2 \frac{\mathbf{s}}{\mathbf{t}}\right), \\
c_{s}^{\prime} & =\frac{2 s+1}{(s-2)(s-1) s(s+1)(s+2)(s+3)} .
\end{aligned}
$$

The u-channel contribution is zero. The total s- plus t-channel contribution to the amplitude from these higher spin exchanges is then

$$
\begin{array}{ll}
A_{s>2}=\frac{\mathbf{u}^{3}}{\mathbf{s}^{2}} \bar{A}\left(\frac{\mathbf{t}}{\mathbf{s}}\right), & \bar{A}(x)=x S(x)+x^{-2} T\left(x^{-1}\right), \\
S(x) \equiv \sum_{s=4,6,8, \ldots}^{\infty} c_{s} P_{s-4}^{(6,2)}(-1-2 x), & T(x) \equiv \sum_{s=3,5,7, \ldots}^{\infty} c_{s}^{\prime} P_{s-4}^{(6,0)}(-1-2 x) .
\end{array}
$$

The explicit evaluation of $S(x)$ and $T(x)$ for $-1<x<0$ gives

$$
\begin{aligned}
& S(x)=-\frac{x^{3}+5 x^{2}+13 x-3}{96(x+1)^{5}}-\frac{x \log (-x)}{8(x+1)^{6}}, \\
& T(x)=-\frac{(x-1)\left(x^{2}+8 x+1\right)}{96(x+1)^{5}}-\frac{x^{2} \log (-x)}{8(x+1)^{6}},
\end{aligned}
$$

\footnotetext{
${ }^{31}$ Here we took into account that for odd spin the momentum space propagator has an extra factor $(-1)^{s}=-1$, cf. $(2.8)$.
} 
so that $\bar{A}=-\frac{1}{96(x+1)}$. The resulting contribution of all higher $s>2$ spin exchanges to the total amplitude is thus

$$
A_{s>2}=-\frac{1}{96} \frac{\mathbf{u}^{3}}{\mathbf{s}^{2}} \frac{1}{\frac{\mathbf{t}}{\mathbf{s}}+1}=\frac{1}{96} \frac{\mathbf{u}^{2}}{\mathbf{s}} .
$$

We are still to add possible contributions of low-spin $s=0,1,2$ exchanges and contact 1122 vertex. The $h_{0}$ exchange is trivial as the 110 vertex (2.11) vanishes for the $\pm 1, \mp 1$ helicity choice. The $h_{2}$ exchange is also found to vanish. The $h_{1}$ exchange gives the following non-zero contributions in the t- and u-channels

$$
\pm 1 \mp 1 \rightarrow \pm 2 \mp 2: \quad A_{\mathbf{t}}^{(1)}=\frac{\mathbf{u}^{3}(2 \mathbf{s}+\mathbf{t})}{192 \mathbf{s}^{3}}, \quad A_{\mathbf{u}}^{(1)}=\frac{\mathbf{t} \mathbf{u}^{2}(\mathbf{s}-\mathbf{t})}{192 \mathbf{s}^{3}} .
$$

The 1122 contact term can be found by computing the UV singular part of the scalar loop diagram with two spin 1 and two spin 2 current insertions
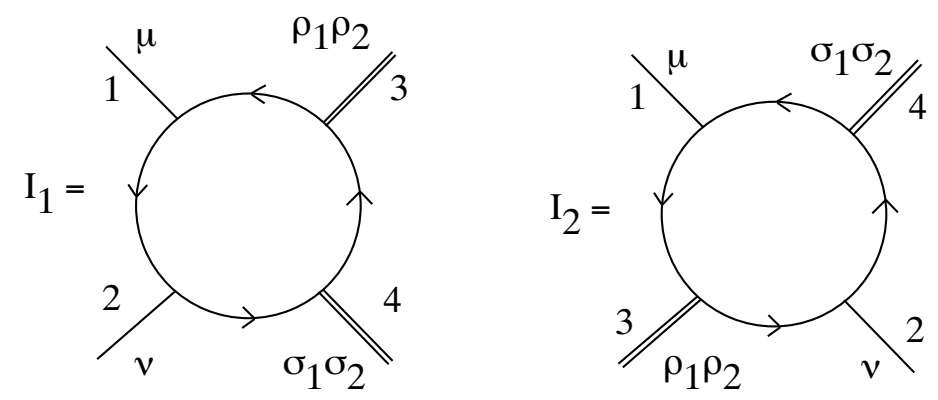

and then replacing the legs with physical polarisations. ${ }^{32}$ We get $I_{1}=\frac{1}{20} \frac{\mathbf{t} \mathbf{u}^{3}}{\mathbf{s}^{3}}, I_{2}=-\frac{1}{60} \frac{\mathbf{t} \mathbf{u}^{3}}{\mathbf{s}^{3}}$. Including combinatorics factors the total contribution is $(-1) \frac{1}{(2 !)^{4}} 2\left(2 I_{1}+I_{2}\right)$, i.e.

$$
\pm 1 \mp 1 \rightarrow \pm 2 \mp 2: \quad A^{(\text {cont })}=-\frac{1}{96} \frac{\mathbf{t} \mathbf{u}^{3}}{\mathbf{s}^{3}} .
$$

The total amplitude given by the sum of the contributions of the higher-spin exchanges (5.23), spin 1 exchange (5.24) and the 4-point contact vertex (5.26) is found to vanish

$$
\pm 1 \mp 1 \rightarrow \pm 2 \mp 2: \quad A_{s>2}+\left[A_{\mathbf{t}}^{(1)}+A_{\mathbf{u}}^{(1)}\right]+A^{(\text {cont })}=0 .
$$

Let us now consider the second non-trivial helicity amplitude $\pm 1 \pm 1 \rightarrow \pm 2 \pm 2$. Here we find that the s-channel amplitude vanishes while the higher odd $s \geq 3$ spin exchange contributions in $\mathrm{t}$ - and $\mathrm{u}$-channels have the form consistent with the general expectations (4.1), (4.5), (4.14)

$$
\begin{aligned}
\pm 1 \pm 1 \rightarrow \pm 2 \pm 2: \quad A_{\mathbf{t}}^{(s)} & =c_{s} \frac{\mathbf{s}^{3}}{\mathbf{t}^{2}} P_{s-3}^{(0,6)}\left(-1-2 \frac{\mathbf{s}}{\mathbf{t}}\right), \quad A_{\mathbf{u}}^{(s)}=c_{s} \frac{\mathbf{s}^{3}}{\mathbf{u}^{2}} P_{s-3}^{(0,6)}\left(-1-2 \frac{\mathbf{s}}{\mathbf{u}}\right) \\
c_{s} & =-\frac{2 s+1}{(s-2)(s-1) s(s+1)(s+2)(s+3)}, \quad s=3,5,7, \ldots
\end{aligned}
$$

The sum over all odd spin $s \geq 3$ exchanges can be done by observing that $P_{s-3}^{(0,6)}(-1-2 x)=$ $P_{s-3}^{(6,0)}(1+2 x)$ and that $c_{s}$ in $(5.28)$ is minus $c_{s}^{\prime}$ in (5.19). One can then use the expression

\footnotetext{
${ }^{32}$ Here the diagrams with the opposite loop orientation give equal contributions.
} 
for $T(x)$ in (5.21), (5.22) to find that the sum of t- and u-channel amplitudes in (5.28) vanishes as a consequence of $\left(x=\frac{\mathbf{s}}{\mathbf{t}}\right)$

$$
x^{2} T(-1-x)+\left(\frac{x}{1+x}\right)^{2} T\left(-\frac{1}{1+x}\right)=0 .
$$

For the non-vanishing low-spin exchange and the 1122 contact term contributions here we get

$$
\begin{aligned}
& \pm 1 \pm 1 \rightarrow \pm 2 \pm 2: \quad A_{\mathbf{s}}^{(0)}=-\frac{\mathbf{s}}{128} \\
& A_{\mathbf{t}}^{(1)}=\frac{\mathbf{u}^{2}\left(\mathbf{s}^{2}-6 \mathbf{s} \mathbf{t}+2 \mathbf{t}^{2}\right)}{128 \mathbf{s}^{3}}, \\
& A_{\mathbf{u}}^{(1)}=\frac{\mathbf{t}^{2}\left(\mathbf{s}^{2}-6 \mathbf{s} \mathbf{u}+2 \mathbf{u}^{2}\right)}{128 \mathbf{s}^{3}}, \quad A^{(\text {cont })}=-\frac{\mathbf{t} \mathbf{u}\left(\mathbf{t}^{2}+3 \mathbf{t} \mathbf{u}+\mathbf{u}^{2}\right)}{32 \mathbf{s}^{3}} .
\end{aligned}
$$

They separately sum up to zero

$$
A_{\mathbf{s}}^{(0)}+\left[A_{\mathbf{t}}^{(1)}+A_{\mathbf{t}}^{(1)}\right]+A^{(\text {cont })}=0,
$$

so the total $\pm 1 \pm 1 \rightarrow \pm 2 \pm 2$ amplitude is again zero.

We conclude that like the $11 \rightarrow 11$ and $22 \rightarrow 22$ amplitudes, the $11 \rightarrow 22$ amplitudes also vanish. It is thus natural to conjecture that all higher spin amplitudes in the CHS theory should also vanish. In appendix B we provide a check of this conjecture by demonstrating that the exchange amplitude for the scattering of four spin $\mathrm{j}$ CHS particles constructed using the general relations in (4.1), (4.5), (4.14) vanishes at the special kinematical point $\mathbf{u}=0$ (i.e. for backward scattering).

\section{Concluding remarks}

In this paper we provided evidence that tree-level 4-particle scattering amplitudes for (massless modes of) conformal higher spin fields vanish after summing over all intermediate CHS exchanges. The amplitudes vanish due to cancellation between the summed up contributions of different scattering channels. This is an indication that this cancellation may be a consequence of the underlying higher spin symmetry which is an infinite dimensional extension of the usual conformal symmetry.

Indeed, the CHS theory inherits the global higher spin symmetry of the free scalar theory (which is also a symmetry of the dual massless higher spin theory in $\mathrm{AdS}_{5}$ ). This symmetry acts on the scalar $\varphi$ and the source fields $h_{s}$ in the coupled action (2.1) and thus becomes the symmetry of the local UV part of the induced action $[4,5] .{ }^{33}$

As was shown in [16], the vanishing of the conformal scalar 4-point amplitude in the coupled scalar - CHS theory can be understood as a consequence of a particular subset of transformations of the higher spin algebra - the hypertranslations $\delta \varphi=\epsilon^{\mu(k)} \partial_{\mu(k)} \varphi$ and the rescalings. Similar reasoning should apply also in the case of the scattering amplitudes with CHS fields on external lines considered in the present paper. The transformation of the CHS fields $h_{s}$ under the differential part of the gauge symmetry is symbolically

\footnotetext{
${ }^{33}$ It also acts on the correlation functions of currents $J_{s}$ at separated points and, vice versa, requiring it to be a symmetry of these correlation functions implies that they should correspond to a free CFT [40-43].
} 
$\delta h=\partial \epsilon+\epsilon \partial h$, i.e. like the usual diffeomorphisms it contains an inhomogeneous and homogeneous parts with the latter mixing different spins. The global part of the algebra corresponds to $\epsilon$ being chosen as conformal Killing tensors. For example, for constant $\epsilon$

$$
\begin{aligned}
\delta h_{0} & =\sum_{k} \epsilon^{\mu(k)} \partial_{\mu(k)} h_{0}, \quad \delta h^{\rho}=\sum_{k}\left[\epsilon^{\rho \mu(k)} \partial_{\mu(k)} h_{0}+\epsilon^{\mu(k)} \partial_{\mu(k)} h^{\rho}\right], \\
\delta h^{\rho \sigma} & =\sum_{k}\left[\epsilon^{\rho \sigma \mu(k)} \partial_{\mu(k)} h_{0}+2 \epsilon^{\mu(k)(\rho} \partial_{\mu(k)} h^{\sigma)}+\epsilon^{\mu(k)} \partial_{\mu(k)} h^{\rho \sigma}\right], \ldots
\end{aligned}
$$

These transformations relate Green's functions with different types of legs $h_{0}, h_{1}, h_{2}, \ldots$. In the case of the S-matrix where the non-propagating field $h_{0}$ does not appear on external lines the transformation of $h_{1}$ under hypertranslations will be the same as of the conformal scalar in [16] so that choosing $\epsilon^{\mu_{1} \ldots \mu_{k}}=y^{\mu_{1}} \ldots y^{\mu_{k}}$ where $y^{\mu}$ is an arbitrary constant vector we may then repeat the argument of [16] for the vanishing of the corresponding scattering amplitude. Similar arguments should also apply to amplitudes involving conformal gravitons.

As the same higher spin algebra controls also the massless higher spin theory in $\mathrm{AdS}_{5}[9]$ (of which the CHS theory is an effective 4d "shadow" or corresponds to the alternative choice of the boundary conditions for higher spin fields [12]) it would be interesting to know if there is an AdS related argument for the vanishing of the CHS S-matrix. One may also start with the CHS theory defined on $\mathrm{AdS}_{4}$ or $\mathrm{dS}_{4}$ and try to generalise the arguments of $[38,39]$ to argue that the corresponding S-matrix for massless higher spin modes of the CHS fields should be the same as in the corresponding massless higher spin theory. Taking the flat limit (i.e. the cosmological constant to zero) may then lead to the conclusion that the S-matrix of the resulting hypothetical massless higher spin theory in flat space should also be trivial.

\section{Acknowledgments}

We would like to thank T. McLoughlin, R. Metsaev, D. Ponomarev and R. Roiban for useful discussions. The work of SN and AAT was supported by the STFC Consolidated grant ST/L00044X/1. The work of AAT was also supported by the ERC Advanced grant No.290456 and by the Russian Science Foundation grant 14-42-00047 associated with Lebedev Institute.

\section{A Vertices in CHS action from scalar loop integrals}

Here we shall provide some details of computation of UV singular parts of the complex scalar loop diagrams with few higher spin current insertions (2.1) leading to the expressions for CHS vertices given in section 2. For the computation of Feynman integrals we shall use the standard relations

$$
\begin{aligned}
\int \frac{d^{d} k}{(2 \pi)^{d}} \frac{\left(k^{2}\right)^{a}}{\left(k^{2}+M^{2}\right)^{b}} & =\frac{\Gamma(b-a-d / 2) \Gamma(a+d / 2)}{(4 \pi)^{d / 2} \Gamma(b) \Gamma(d / 2)}\left(M^{2}\right)^{d / 2+a-b}, \\
\frac{1}{A_{1} \ldots A_{n}} & =(n-1) ! \int_{[0,1]^{n}} d^{n} x \frac{\delta\left(x_{1}+\cdots+x_{n}-1\right)}{\left(x_{1} A_{1}+\cdots+x_{n} A_{n}\right)^{n}} .
\end{aligned}
$$


The expression for the scalar loop diagram in (2.7) with two current operator or vertex (2.6) insertions has the following general structure

$$
\begin{aligned}
\int \frac{d^{d} k}{(2 \pi)^{d}} \frac{N(k, p)}{k^{2}(k+p)^{2}} & =\int_{0}^{1} d x \int \frac{d^{d} k}{(2 \pi)^{d}} \frac{N(k, p)}{\left[(k+x p)^{2}+x(1-x) p^{2}\right]^{2}} \\
& =\int_{0}^{1} d x \int \frac{d^{d} k}{(2 \pi)^{d}} \frac{N(k-x p, p)}{\left(k^{2}+M^{2}\right)^{2}}, \quad M^{2}=x(1-x) p^{2} .
\end{aligned}
$$

When contracted with two TT fields $h_{s}$ the numerator in (A.3) takes the following form

$$
N_{\mu(s) \nu(s)}(k-p x, p) \rightarrow \frac{1}{(s !)^{2}} k_{\mu_{1}} k_{\nu_{1}} \ldots k_{\mu_{s}} k_{\nu_{s}} \rightarrow \frac{1}{(s !)^{2}} \frac{1}{2^{s}(s+1)}\left(k^{2}\right)^{s} \eta_{\mu_{1} \nu_{1}} \cdots \eta_{\mu_{s} \nu_{s}} .
$$

Then integrating over $k$ and extracting the coefficient of the $\frac{1}{\epsilon}$ pole term we find

$$
S_{2}\left[h_{s}\right]=\frac{(-1)^{s}}{2^{s} \Gamma(2 s+2)} \int \frac{d^{4} p}{(2 \pi)^{4}} h_{\mu(s)}(p)\left(p^{2}\right)^{s} h^{\mu(s)}(-p),
$$

which becomes (2.8) when written in coordinate representation.

The cubic vertex for three CHS fields is determined by the diagram in (2.9). For the 1-1-s vertex multiplied by $h_{\mu}\left(p_{1}\right) h_{\nu}\left(p_{2}\right) h_{\rho(s)}\left(-p_{1}-p_{2}\right)$ in the CHS action we get $^{34}$

$$
V_{\mu, \nu, \rho(s)}\left(p_{1}, p_{2}\right)=\frac{1}{2 !} \frac{1}{s !} \int \frac{d^{d} k}{(2 \pi)^{d}} \frac{k_{\mu}\left(k+p_{1}\right)_{\nu}\left(k+p_{1}+p_{2}\right)_{\rho(s)}}{k^{2}\left(k+p_{1}\right)^{2}\left(k+p_{1}+p_{2}\right)^{2}} .
$$

There is also another diagram with the scalar $\mathrm{U}(1)$ charge flowing in the opposite direction giving $\widetilde{V}_{\mu, \nu, r h o(s)}\left(p_{1}, p_{2}\right)=V_{\nu, \mu, \rho(s)}\left(p_{2}, p_{1}\right)$; their sum

$$
\mathrm{V}_{\mu, \nu, \rho(s)}\left(p_{1}, p_{2}\right)=V_{\mu, \nu, \rho(s)}\left(p_{1}, p_{2}\right)+V_{\nu, \mu, \rho(s)}\left(p_{2}, p_{1}\right)
$$

ensures the symmetry under $h_{\mu}\left(p_{1}\right) \leftrightarrow h_{\nu}\left(p_{2}\right)$. To compute the pole part of this integral we use Feynman parametrisation (and shifts of $k$ ) and assume that the external legs are contracted with TT fields (i.e. terms with $p_{1 \mu}, p_{2 \nu},\left(p_{1}+p_{2}\right)_{\rho(s)}$ can be dropped). Then

$$
\begin{aligned}
V_{\mu, \nu, \rho(s)} & =\frac{1}{2 s !} \int \frac{d^{d} k}{(2 \pi)^{d}} \frac{k_{\mu} k_{\rho(s)}\left(k+p_{1}\right)_{\nu}}{k^{2}\left(k+p_{1}\right)^{2}\left(k+p_{1}+p_{2}\right)^{2}} \\
& =\frac{1}{s !} \int_{0}^{1} d x \int_{0}^{1-x} d y \int \frac{d^{d} k}{(2 \pi)^{d}} \frac{k_{\mu} k_{\rho(s)}\left(k+p_{1}\right)_{\nu}}{\left[\left(k+x p_{1}+y\left(p_{1}+p_{2}\right)\right)^{2}+M^{2}\right]^{3}} \\
& \rightarrow \frac{1}{s !} \int_{0}^{1} d x \int_{0}^{1-x} d y \int \frac{d^{d} k}{(2 \pi)^{d}} \frac{\left(k-y p_{2}\right)_{\mu}\left(k-x p_{1}\right)_{\rho(s)}\left(k+(1-x-y) p_{1}\right)_{\nu}}{\left(k^{2}+M^{2}\right)^{3}} \\
M^{2} & =x(1-x) p_{1}^{2}+y(1-y)\left(p_{1}+p_{2}\right)^{2}-2 x y p_{1} \cdot\left(p_{1}+p_{2}\right) .
\end{aligned}
$$

Separating terms of different order in $k$ in the numerator, integrating over $k$ and then over $x, y$ we find for the pole part

$$
\begin{aligned}
V_{\mu, \nu, \rho(s)}\left(p_{1}, p_{2}\right)=\frac{1}{2(s+2) !}\{ & \eta_{\mu \nu}\left(p_{1}\right)_{\rho(s)}-\eta_{\mu \rho_{1}} p_{1 \nu} p_{1 \rho_{2}} \ldots p_{1 \rho_{s}}+\eta_{\nu \rho_{1}} p_{2 \mu} p_{1 \rho_{2}} \ldots p_{1 \rho_{s}} \\
- & \left.\eta_{\mu \rho_{1}} \eta_{\nu \rho_{2}} p_{1 \rho_{3}} \ldots p_{1 \rho_{s}}\left[p_{1} \cdot p_{2}+\frac{s}{2}\left(p_{1}^{2}+p_{2}^{2}\right)\right]\right\} . \quad \text { A }
\end{aligned}
$$

\footnotetext{
${ }^{34}$ Here only the UV pole part is to be kept: for simplicity, here and below we shall use use the same notation $V$ for the full integral and the coefficient of its pole part, i.e. $V \rightarrow \frac{1}{(4 \pi)^{2} \epsilon} V+$ finite.
} 
The full cubic 1-1-s vertex is then given by (3.4) or (2.10) in coordinate representation.

The quartic 1111 vertex (2.13) is found from the pole part of the diagram in (2.12)

$$
\begin{aligned}
& \frac{1}{4 !} \times 6 \times 3 ! \int_{0}^{1} d x \int_{0}^{1-x} d y \int_{0}^{1-x-y} d z \int \frac{d^{d} k}{(2 \pi)^{d}} \frac{k_{\mu} k_{\nu} k_{\rho} k_{\sigma}}{\left(k^{2}+M^{2}\right)^{4}} \\
& \rightarrow \frac{1}{16 \pi^{2} \epsilon} \frac{1}{48}\left(\eta_{\mu \nu} \eta_{\rho \sigma}+\eta_{\mu \rho} \eta_{\nu \sigma}+\eta_{\mu \sigma} \eta_{\nu \rho}\right) .
\end{aligned}
$$

To find the 2-2-s vertex which is multiplied by the TT fields $h_{\mu_{1} \mu_{2}}\left(p_{1}\right) h_{\nu_{1} \nu_{2}}\left(p_{2}\right) h_{\rho(s)}$ in the CHS action we are to find again the singular part of the diagram (2.9) with the vertices (2.6) leading to the integral (where we are allowed to drop TT-trivial terms proportional to $p_{1 \mu_{1}}, p_{1 \mu_{2}}$, etc.)

$$
\begin{aligned}
V_{\mu_{1} \mu_{2}, \nu_{1} \nu_{2}, \rho(s)} & =\frac{1}{2 !} \frac{1}{(2 !)^{2}} \frac{1}{s !} \int \frac{d^{d} k}{(2 \pi)^{d}} \frac{k_{\mu_{1}} k_{\mu_{2}}\left(k+p_{1}\right)_{\nu_{1}}\left(k+p_{1}\right)_{\nu_{2}}\left(k+p_{1}+p_{2}\right)_{\rho(s)}}{k^{2}\left(k+p_{1}\right)^{2}\left(k+p_{1}+p_{2}\right)^{2}} \\
& \rightarrow \frac{1}{4 s !} \int_{0}^{1} d x \int_{0}^{1-x} d y \int \frac{d^{d} k}{(2 \pi)^{d}} \frac{k_{\mu_{1}} k_{\mu_{2}} k_{\rho(s)}\left(k+p_{1}\right)_{\nu_{1}}\left(k+p_{1}\right)_{\nu_{2}}}{\left[\left(k+x p_{1}+y\left(p_{1}+p_{2}\right)\right)^{2}+M^{2}\right]^{3}} \\
& \rightarrow \frac{1}{4 s !} \int_{0}^{1} d x \int_{0}^{1-x} d y \int \frac{d^{d} k}{(2 \pi)^{d}} \frac{N_{\mu_{1} \mu_{2}, \nu_{1} \nu_{2}, \rho(s)}\left(p_{1}, p_{2}, k ; x, y\right)}{\left(k^{2}+M^{2}\right)^{3}}
\end{aligned}
$$

where $M^{2}$ is the same as in (A.9) and

$N_{\mu_{1} \mu_{2}, \nu_{1} \nu_{2}, \rho(s)}=\left(k-y p_{2}\right)_{\mu_{1}}\left(k-y p_{2}\right)_{\mu_{2}}\left(k-x p_{1}\right)_{\rho(s)}\left[k+(1-x-y) p_{1}\right]_{\nu_{1}}\left[k+(1-x-y) p_{1}\right]_{\nu_{2}}$

Non-trivial UV divergent pole contributions may come from the terms in $N$ which are of order $k^{2}, k^{4}, k^{6}, k^{8}$ (integrals of higher powers of $k$ will lead to contractions between $\rho$ indices that can be discarded due to TT condition). As a result, we find the vertex given in (2.15).

Let us also discuss some vertices involving the non-propagating spin 0 field $h_{0}$. One can show that 0-0-s interaction is absent if $h_{s}$ is subject to TT condition. 1-0-s vertex is given by the pole part of the integral (here $p=p_{2}$ and $M^{2}$ is as in (A.9))

$$
\mathrm{V}_{\mu, \rho(s)}=\frac{4}{s !} \int_{0}^{1} d x \int_{0}^{1-x} d y \int \frac{d^{d} k}{(2 \pi)^{d}} \frac{(k-y p)_{\mu}(k+x p)_{\rho(s)}}{\left(k^{2}+M^{2}\right)^{3}}
$$

It is non-vanishing for odd $s$ and reduces to (2.17). In the case of 2-0-s vertex we get two diagrams (2.9) with opposite loop direction and the sum of the corresponding integrals can be put into the form

$$
\begin{aligned}
\mathrm{V}_{\mu_{1} \mu_{2}, \rho(s)} & =\frac{1}{s !} \int \frac{d^{d} k}{(2 \pi)^{d}} \frac{k_{\mu_{1}} k_{\mu_{2}} k_{\rho(s)}}{k^{2}\left(k+p_{1}\right)^{2}\left(k+p_{1}+p_{2}\right)^{2}} \\
& \rightarrow \frac{2}{s !} \int_{0}^{1} d x \int_{0}^{1-x} d y \int \frac{d^{d} k}{(2 \pi)^{d}} \frac{k_{\mu_{1}} k_{\mu_{2}} k_{\rho(s)}}{\left[\left(k+x p_{1}+y\left(p_{1}+p_{2}\right)\right)^{2}+M^{2}\right]^{3}} \\
& \rightarrow \frac{2}{s !} \int_{0}^{1} d x \int_{0}^{1-x} d y \int \frac{d^{d} k}{(2 \pi)^{d}} \frac{(k-y p)_{\mu_{1}}(k-y p)_{\mu_{2}}(k+x p)_{\rho(s)}}{\left(k^{2}+M^{2}\right)^{3}}
\end{aligned}
$$

where $M^{2}$ is as in (A.9). The pole part of this integral is given by (2.18). The computation of the 1-2-s vertex is similar, leading to the expression in (2.19). 


\section{B Vanishing of 4-particle amplitude at special kinematics}

\section{B.1 $11 \rightarrow 11$ scattering}

As a check of the vanishing of the summed over spins $11 \rightarrow 11$ scattering amplitude discussed in section 3.2 here we independently demonstrate this at the special kinematics point $\mathbf{u}=0$ or $\mathbf{s}=-\mathbf{t}$ corresponding to $\theta=\pi$ or backward scattering. The total $++\rightarrow++$ amplitude obtained by summing (3.18) over all even spins $s=2,4, \ldots$ for $\mathbf{s}=-\mathbf{t}$ may be written as

$$
\left.\sum_{s=2,4, \ldots}^{\infty} A^{(s)}\right|_{\mathbf{u} \rightarrow 0}=a+\lim _{\gamma \rightarrow \infty} f(\gamma), \quad a=\sum_{s=2,4, \ldots}^{\infty} c_{s}, \quad f(\gamma)=\sum_{s=2,4, \ldots}^{\infty} c_{s} \gamma^{s} P_{s}(0),
$$

where the first term comes from the t-channel and the second from the u-channel contribution (where we already set $\mathbf{u}=0$ in the argument of $P_{s}$ ). Here we defined $\gamma \equiv \frac{\mathbf{s}}{\mathbf{u}} \rightarrow \infty$ and used that according to (3.11) $P_{s}(-1)=1$. From (3.12) we have

$$
a=\sum_{s=2,4, \ldots}^{\infty} \frac{2 s+1}{2(s-1) s(s+1)(s+2)}=\frac{1}{8} .
$$

From (3.11) and (3.12) we get

$$
c_{s} P_{s}(0)=\frac{\Gamma(2 s+2)}{2[\Gamma(s+3)]^{2}},
$$

and then find that

$$
\begin{aligned}
f(\gamma)=-\frac{1}{48 \gamma^{2}}[ & 6 \gamma^{2}{ }_{4} F_{3}\left(\frac{3}{4}, 1,1, \frac{5}{4} ; \frac{3}{2}, 2,2 ; 16 \gamma^{2}\right)+160 \gamma^{4}{ }_{3} F_{2}\left(\frac{3}{2}, \frac{7}{4}, \frac{9}{4} ; \frac{5}{2}, \frac{5}{2} ; 16 \gamma^{2}\right) \\
& \left.-3 \sqrt{2} \sqrt{\sqrt{1-16 \gamma^{2}}+1}+6\left(\gamma-\frac{1}{\sqrt{1-4 \gamma}}+\frac{1}{\sqrt{4 \gamma+1}}\right) \gamma+6\right] .
\end{aligned}
$$

For small $\gamma$ we get the expansion $f(\gamma)=\frac{5 \gamma^{2}}{48}+\frac{7 \gamma^{4}}{20}+\frac{429 \gamma^{6}}{224}+\ldots$ which is convergent for $|\gamma|<\frac{1}{4}$. Using the analytic continuation, we may extend $f(\gamma)$ beyond this convergence disk and evaluate it at $\gamma \rightarrow \infty$, getting

$$
\lim _{\gamma \rightarrow \infty} f(\gamma)=-\frac{1}{8}
$$

As a result, we conclude that the $\mathbf{u} \rightarrow 0$ limits of the t-channel (B.2) and u-channel (B.5) contributions indeed cancel against each other just as was found for general kinematics in $(3.25)$.

\section{B.2 $\mathrm{j} \mathrm{j} \rightarrow \mathrm{j}$ j scattering}

To check our conjecture that all 4-point CHS amplitudes should vanish we may (i) first make a guess for the CHS spin $\mathrm{j}$ 4-point exchange amplitude generalising the expressions for the $11 \rightarrow 11$ and $22 \rightarrow 22$ amplitudes explicitly computed in (3.18), (3.11) and (5.2), (5.3) being 
guided by the expected structure of spin $J \geq 2 \mathrm{j}$ exchange amplitude in (4.1), (4.5), (4.14) and (ii) then check its vanishing at a special kinematical point. ${ }^{35}$

Then the total amplitude is expected to be given as in (3.19), (3.25) and (5.6), (5.9) by the sum of the t-channel and u-channel exchanges of even spin $s$ CHS states

$$
\begin{aligned}
A & =\mathbf{s}^{2 \mathrm{j}-2}[\sigma(x)+\sigma(-1-x)], \quad x=\frac{\mathbf{t}}{\mathbf{s}}, \\
\sigma(x) & =\frac{2}{x^{2}} \sum_{J=2 \mathrm{j}, 2 \mathrm{j}+2, \ldots}^{\infty}\left(J+\frac{1}{2}\right) \frac{(J-2 \mathrm{j}) !}{(J+2 \mathrm{j}) !} P_{J-2 \mathrm{j}}^{(4 \mathrm{j}, 0)}\left(\frac{x+2}{x}\right) \\
& =\frac{2}{x^{2}} \sum_{s=0,2,4, \ldots}^{\infty}\left(s+2 \mathrm{j}+\frac{1}{2}\right) \frac{s !}{(s+4 \mathrm{j}) !} P_{s}^{(4 \mathrm{j}, 0)}\left(\frac{x+2}{x}\right) .
\end{aligned}
$$

Let us now show the vanishing of the sum $\sigma(x)+\sigma(-1-x)$ at $\mathbf{u}=0$ or $x=\frac{\mathbf{t}}{\mathbf{s}}=-1$, i.e. $\sigma(0)=-\sigma(-1)$. This is equivalent also to proving the vanishing of this sum at $x=0 .{ }^{36}$

Using that $P_{s}^{(4 \mathrm{j}, 0)}(-1)=1$ we get $^{37}$

$$
\begin{aligned}
& \sigma(-1)=\sum_{s=0,2,4, \ldots}^{\infty}(2 s+4 \mathrm{j}+1) \frac{s !}{(s+4 \mathrm{j}) !} \\
& =\sqrt{\pi} 2^{-4 \mathrm{j}}\left[(4 \mathrm{j}+1)_{3} \widetilde{F}_{2}\left[\begin{array}{c}
\frac{1}{2} 11 \\
2 \mathrm{j}+\frac{1}{2} 2 \mathrm{j}+1
\end{array} ; 1\right]+2{ }_{3} \widetilde{F}_{2}\left[\begin{array}{c}
\frac{3}{2} 2 \\
2 \mathrm{j}+\frac{3}{2} 2 \mathrm{j}+2
\end{array} ; 1\right]\right] \\
& =\frac{1}{4(2 \mathrm{j}-1)^{2} \Gamma(4 \mathrm{j}-2)} \text {. }
\end{aligned}
$$

To compute $\sigma(0)$ we note that for $x \rightarrow 0$ the leading term in the expansion of the Jacobi polynomial is

$$
\left.P_{s}^{(4 \mathrm{j}, 0)}\left(\frac{x+2}{x}\right)\right|_{x \rightarrow 0}=\frac{1}{x^{s}} \frac{4^{s+2 \mathrm{j}}}{\sqrt{\pi}} \frac{(s+2 \mathrm{j}) !}{s !} \frac{\Gamma\left(s+2 \mathrm{j}+\frac{1}{2}\right)}{\Gamma(s+4 \mathrm{j}+1)}+\cdots
$$

Plugging this into (B.7) and taking the $x \rightarrow 0$ limit we get

$$
\begin{aligned}
\sigma(0)=\lim _{x \rightarrow 0}\left[\frac{1}{\Gamma(4 \mathrm{j})} x^{-2}{ }_{5} F_{4}\left[\begin{array}{c}
1 \mathrm{j}+\frac{1}{4} \mathrm{j}+\frac{1}{2} \mathrm{j}+\frac{3}{4} \mathrm{j}+1 \\
2 \mathrm{j}+\frac{1}{2} 2 \mathrm{j}+\frac{1}{2} 2 \mathrm{j}+12 \mathrm{j}+1 ; \frac{16}{x^{2}}
\end{array}\right]\right. \\
\left.+\frac{4^{2 \mathrm{j}+3} \Gamma\left(2 \mathrm{j}+\frac{5}{2}\right) \Gamma(2 \mathrm{j}+3)}{\sqrt{\pi} \Gamma(4 \mathrm{j}+3)} x^{-4}{ }_{5} F_{4}\left[\begin{array}{c}
2 \mathrm{j}+\frac{5}{4} \mathrm{j}+\frac{3}{2} \mathrm{j}+\frac{7}{4} \mathrm{j}+2 \\
2 \mathrm{j}+\frac{3}{2} 2 \mathrm{j}+\frac{3}{2} 2 \mathrm{j}+22 \mathrm{j}+2
\end{array} ; \frac{16}{x^{2}}\right]\right]
\end{aligned}
$$

\footnotetext{
${ }^{35}$ We shall assume that as in the $\mathrm{j}=1$ and $\mathrm{j}=2$ cases (see section 3.2 and (5.13)) the sum of the low-spin $J<2 \mathrm{j}$ exchanges and contact jjjj contribution vanishes separately.

${ }^{36}$ Notice that it is not possible to check numerically the vanishing of the amplitude $A$ in (B.6) at a generic value of the kinematical variable $x$. This is because the series in (B.7) converges for $x \leq-1$ and a test of the condition $A=0$ requires the analytical continuation of the series definition of $\sigma(x)$. The explicit summation over the spin $s$ leads to the result which has an expected non-trivial analytical structure with branch points at $x=0,1$, see, for instance, (3.24) and (5.8).

${ }^{37}$ Here ${ }_{p} \widetilde{F}_{q}\left[\begin{array}{c}a_{1} \ldots a_{n} \\ b_{1} \ldots b_{m}\end{array} ; z\right]=\frac{1}{\Gamma\left(b_{1}\right) \ldots \Gamma\left(b_{m}\right)}{ }_{p} F_{q}\left[\begin{array}{c}a_{1} \ldots a_{n} \\ b_{1} \ldots b_{m}\end{array} ; z\right]$ is the regularised hypergeometric function. We use the compact notation ${ }_{p} F_{q}\left[\begin{array}{c}a_{1} \ldots a_{n} \\ b_{1} \ldots b_{m}\end{array} ;\right] \equiv{ }_{p} F_{q}\left(a_{1}, \ldots, a_{n} ; b_{1}, \ldots, b_{m} ; z\right)$ for the generalised hypergeometric function.
} 
This $x \rightarrow 0$ limit exists and can be explicitly evaluated for $\mathrm{j}=1,2,3, \ldots$. One finds that the resulting value of (B.10) is minus that of (B.8), ${ }^{38}$ i.e. $\sigma(0)=-\sigma(-1)$. Thus the total amplitude (B.6) vanishes at $\mathbf{u}=0$ (or $\mathbf{t}=0$ ).

\section{Derivation of the general form of the $11 \rightarrow 11$ spin $s$ exchange ampli- tude}

Our starting point is the 1-1-s vertex in (3.4), (A.10) that may be written as

$$
\begin{aligned}
\mathrm{V}^{\mu, \nu, \rho(s)}(p, q) & =\frac{1}{(s+2) !} q^{\rho_{3}} \ldots q^{\rho_{s}} \hat{V}^{\mu, \nu, \rho_{1} \rho_{2}}(p, q), \\
\hat{V}^{\mu, \nu, \rho_{1} \rho_{2}}(p, q) & =\eta^{\mu \nu} q^{\rho_{1}} q^{\rho_{2}}-\eta^{\rho_{1} \mu} q^{\nu} q^{\rho_{2}}+\eta^{\rho_{1} \nu} p^{\mu} q^{\rho_{2}}-\left[p \cdot q+\frac{s}{2}\left(p^{2}+q^{2}\right)\right] \eta^{\mu \rho_{1}} \eta^{\nu \rho_{2}},
\end{aligned}
$$

where symmetrisation over $\rho_{1}, \ldots, \rho_{s}$ is assumed. Let us contract the $\rho_{i}$ indices with an auxiliary vector $u^{\rho}$, i.e. define

$$
\begin{aligned}
& V^{\mu, \nu}(p, q, u)=\frac{1}{2(s+2) !}(q \cdot u)^{s-2} \hat{V}^{\mu, \nu}(p, q, u), \\
& \hat{V}^{\mu, \nu}(p, q, u)=\eta^{\mu \nu}(q \cdot u)^{2}-\left(u^{\mu} q^{\nu}-u^{\nu} p^{\mu}\right) q \cdot u+-\left[p \cdot q+\frac{s}{2}\left(p^{2}+q^{2}\right)\right] u^{\mu} u^{\nu} .
\end{aligned}
$$

The TT projector in the CHS propagator (3.5) acting on monomials of $u$ may be written as

$$
\begin{aligned}
\Pi^{(s)}\left(\partial_{u_{1}}, \partial_{u_{2}}, k\right) & =\frac{1}{(s !)^{2}} \sum_{l=0}^{[s / 2]} b_{s, l} Y_{1}^{l} Y_{2}^{l} X^{s-2 l}, & a_{s, l} & =(-1)^{l} \frac{s ! \Gamma\left(s-l+\frac{1}{2}\right)}{2^{2 l} l !(s-2 l) ! \Gamma\left(s+\frac{1}{2}\right)}, \\
X & =\partial_{u_{1}} \cdot \partial_{u_{2}}-\frac{\left(\partial_{u_{1}} \cdot k\right)\left(\partial_{u_{2}} \cdot k\right)}{k^{2}}, & Y_{i} & =\partial_{u_{i}}^{2}-\frac{\left(\partial_{u_{i}} \cdot k\right)\left(\partial_{u_{i}} \cdot k\right)}{k^{2}} .
\end{aligned}
$$

To compute the exchange amplitude, e.g., in the case of $+-\rightarrow+-$ scattering in s-channel (cf. (3.8)) we need to contract two vertices with CHS propagator, i.e. compute

$$
\left.\Pi^{(s)}\left(\partial_{u_{1}}, \partial_{u_{2}}, p_{1}+p_{2}\right) V^{\mu_{1}, \mu_{2}}\left(p_{1}, p_{2}, u_{1}\right) V^{\nu_{1}, \nu_{2}}\left(p_{3}, p_{4}, u_{2}\right)\right|_{u_{i}=0}
$$

Using (C.4) the $s$ dependence is determined by

$$
\left.\Pi_{2}^{(s)} \equiv[s(s-1)]^{2} \Pi^{(s)}\left(\partial_{u_{1}}, \partial_{u_{2}}, p_{1}+p_{2}\right)\left(p_{2} \cdot u_{1}\right)^{s-2}\left(p_{4} \cdot u_{2}\right)^{s-2}\right|_{u_{i}=0} .
$$

Here the subscript denotes that this is a differential operator of order 2 in $\partial_{u_{1}}$ and $\partial_{u_{2}}$. Let us introduce:

$$
\begin{aligned}
W_{1} & =p_{4} \cdot \partial_{u_{1}}+\frac{1}{2}\left(p_{1}+p_{2}\right) \cdot \partial_{u_{1}}, & W_{2} & =p_{2} \cdot \partial_{u_{2}}-\frac{1}{2}\left(p_{1}+p_{2}\right) \cdot \partial_{u_{2}}, \\
Z_{1} & =2 p_{2} \cdot \partial_{u_{1}}-\left(p_{1}+p_{2}\right) \cdot \partial_{u_{1}}, & Z_{2} & =2 p_{4} \cdot \partial_{u_{2}}+\left(p_{1}+p_{2}\right) \cdot \partial_{u_{2}}
\end{aligned}
$$

\footnotetext{
${ }^{38}$ It should be possible to prove this fact analytically given the simplicity of the result in (B.8).
} 
so that we get

$$
\begin{aligned}
{\left[X,\left(u_{1} \cdot p_{2}\right)\left(u_{2} \cdot p_{4}\right)\right] } & =\tilde{\mathbf{t}}+\left(p_{2} \cdot u_{1}\right) W_{2}+\left(p_{4} \cdot u_{2}\right) W_{1}, \\
{\left[Y_{1},\left(u_{1} \cdot p_{2}\right)^{2}\right] } & =\tilde{\mathbf{s}}+\left(p_{2} \cdot u_{1}\right) Z_{1}, \quad\left[Y_{2},\left(p_{4} \cdot u_{2}\right)^{2}\right]=\tilde{\mathbf{s}}+\left(p_{4} \cdot u_{2}\right) Z_{2} \quad(C .7) \\
{\left[Z_{1},\left(p_{2} \cdot u_{1}\right)\right] } & =\tilde{\mathbf{s}}, \quad\left[Z_{2},\left(p_{4} \cdot u_{2}\right)\right]=\tilde{\mathbf{s}}, \quad\left[W_{1},\left(p_{2} \cdot u_{1}\right)\right]=\tilde{\mathbf{t}}, \quad\left[W_{2},\left(p_{4} \cdot u_{2}\right)\right]=\tilde{\mathbf{t}}
\end{aligned}
$$

where $\tilde{\mathbf{s}} \equiv-\frac{\mathbf{s}}{4}$ and $\tilde{\mathbf{t}} \equiv \frac{1}{2}\left(\mathbf{t}+\frac{\mathbf{s}}{2}\right)$. Then we may commute $Y_{1}^{l} Y_{2}^{l} X^{s-2 l}$ with $\left(p_{2} \cdot u_{1}\right)^{s-2}\left(p_{4} \cdot u_{2}\right)^{s-2}$ and the result will be composed of the operators $Y_{1}, Y_{2}, X, W_{1}, W_{2}, Z_{1}$ and $Z_{2}$. A generating function determining the combinatoric coefficient is

$$
\begin{aligned}
\mathcal{P}^{(s)}=\sum_{j=0}^{\infty}\left(t_{1} t_{2}\right)^{j} \Pi_{j}^{(s)}= & \sum_{l=0}^{\left[\frac{s}{2}\right]} a_{s, l} \tilde{\mathbf{t}}^{s-2 l} \tilde{\mathbf{s}}^{2 l}\left[1+\tilde{\mathbf{t}}^{-1}\left(t_{1} W_{1}+t_{2} W_{2}+t_{1} t_{2} X\right)\right]^{s-2 l} \\
\times & {\left[1+\tilde{\mathbf{s}}^{-1}\left(t_{1} Z_{1}+t_{1}^{2} Y_{1}\right)\right]^{l}\left[1+\tilde{\mathbf{s}}^{-1}\left(t_{2} Z_{2}+t_{2}^{2} Y_{2}\right)\right]^{l} . }
\end{aligned}
$$

Using this to compute (C.5) and thus (C.4) we may get an expression for the amplitude in terms of a hypergeometric function of the kinematic variables. Adding the s-channel propagator $\mathbf{s}^{-s}$ factor in (3.5) the result for the $+-\rightarrow+-$ exchange amplitude in the s-channel may be written as

$$
A_{\mathbf{s}+,-;+,-}^{(s)}=c_{s} x^{-2} P_{s-2}^{(4,0)}\left(\frac{x+2}{x}\right), \quad c_{s}=2(2 s+1) \frac{(s-2) !}{(s+2) !}, \quad x=\frac{\mathbf{s}}{\mathbf{u}},
$$

in agreement with (3.11), (3.12).

Similar derivation may be given for the spin $s$ exchange contribution to the $22 \rightarrow 22$ amplitude. Here we use that the 2-2-s vertex (2.15) may be written as

$$
\mathrm{V}^{\mu_{1} \mu_{2}, \nu_{1} \nu_{2}, \rho(s)}(p, q)=\frac{1}{8(s+4) !} q^{\rho_{5}} \ldots q^{\rho_{s}} \hat{V}^{\mu_{1} \mu_{2}, \nu_{1} \nu_{2}, \rho(4)}(p, q),
$$

or, when contracted with $u^{\rho}$,

$$
\mathrm{V}^{\mu_{1} \mu_{2}, \nu_{1} \nu_{2}, \rho(s)}(p, q, u)=\frac{1}{8(s+4) !}(q \cdot u)^{s-4} \hat{V}^{\mu_{1} \mu_{2}, \nu_{1} \nu_{2}, \rho(4)}(p, q, u) .
$$

Here we need to find the $\Pi_{4}^{(s)}$ analog of $\Pi_{2}^{(s)}$ in (C.5) and it can be readily obtained from (C.8). The final result matches the expressions in (5.2)-(5.5).

Open Access. This article is distributed under the terms of the Creative Commons Attribution License (CC-BY 4.0), which permits any use, distribution and reproduction in any medium, provided the original author(s) and source are credited.

\section{References}

[1] E.S. Fradkin and A.A. Tseytlin, Conformal supergravity, Phys. Rept. 119 (1985) 233 [INSPIRE]. 
[2] E.S. Fradkin and V. Ya. Linetsky, Cubic interaction in conformal theory of integer higher spin fields in four-dimensional space-time, Phys. Lett. B 231 (1989) 97 [INSPIRE].

[3] A.A. Tseytlin, On limits of superstring in $A d S_{5} \times S^{5}$, Theor. Math. Phys. 133 (2002) 1376 [Teor. Mat. Fiz. 133 (2002) 69] [hep-th/0201112] [INSPIRE].

[4] A.Y. Segal, Conformal higher spin theory, Nucl. Phys. B 664 (2003) 59 [hep-th/0207212] [INSPIRE].

[5] X. Bekaert, E. Joung and J. Mourad, Effective action in a higher-spin background, JHEP 02 (2011) 048 [arXiv: 1012.2103] [INSPIRE].

[6] M. Beccaria, X. Bekaert and A.A. Tseytlin, Partition function of free conformal higher spin theory, JHEP 08 (2014) 113 [arXiv: 1406.3542] [INSPIRE].

[7] P. Haehnel and T. McLoughlin, Conformal higher spin theory and twistor space actions, arXiv: 1604.08209 [INSPIRE].

[8] M.G. Eastwood, Higher symmetries of the Laplacian, Annals Math. 161 (2005) 1645 [hep-th/0206233] [INSPIRE].

[9] M.A. Vasiliev, Nonlinear equations for symmetric massless higher spin fields in $(A) d S_{d}$, Phys. Lett. B 567 (2003) 139 [hep-th/0304049] [INSPIRE].

[10] I.R. Klebanov and A.M. Polyakov, AdS dual of the critical $O(N)$ vector model, Phys. Lett. B 550 (2002) 213 [hep-th/0210114] [INSPIRE].

[11] M. Beccaria and A.A. Tseytlin, On higher spin partition functions, J. Phys. A 48 (2015) 275401 [arXiv: 1503.08143] [INSPIRE].

[12] S. Giombi, I.R. Klebanov, S.S. Pufu, B.R. Safdi and G. Tarnopolsky, AdS description of induced higher-spin gauge theory, JHEP 10 (2013) 016 [arXiv:1306.5242] [INSPIRE].

[13] A.A. Tseytlin, On partition function and Weyl anomaly of conformal higher spin fields, Nucl. Phys. B 877 (2013) 598 [arXiv: 1309.0785] [InSPIRE].

[14] M. Beccaria and A.A. Tseytlin, Higher spins in AdS $S_{5}$ at one loop: vacuum energy, boundary conformal anomalies and AdS/CFT, JHEP 11 (2014) 114 [arXiv:1410.3273] [INSPIRE].

[15] S. Giombi, I.R. Klebanov and B.R. Safdi, Higher spin $A d S_{d+1} / C F T_{d}$ at one loop, Phys. Rev. D 89 (2014) 084004 [arXiv: 1401.0825] [INSPIRE].

[16] E. Joung, S. Nakach and A.A. Tseytlin, Scalar scattering via conformal higher spin exchange, JHEP 02 (2016) 125 [arXiv: 1512.08896] [INSPIRE].

[17] M. Jacob and G.C. Wick, On the general theory of collisions for particles with spin, Annals Phys. 7 (1959) 404 [INSPIRE].

[18] D.Z. Freedman, K. Johnson and J.I. Latorre, Differential regularization and renormalization: a new method of calculation in quantum field theory, Nucl. Phys. B 371 (1992) 353 [INSPIRE].

[19] R.R. Metsaev, Ordinary-derivative formulation of conformal low spin fields, JHEP 01 (2012) 064 [arXiv: 0707.4437] [INSPIRE].

[20] R.R. Metsaev, Ordinary-derivative formulation of conformal totally symmetric arbitrary spin bosonic fields, JHEP 06 (2012) 062 [arXiv:0709.4392] [INSPIRE].

[21] H. Elvang and Y.-T. Huang, Scattering amplitudes, arXiv:1308.1697 [INSPIRE].

[22] T. Gleisberg, F. Krauss, K.T. Matchev, A. Schalicke, S. Schumann and G. Soff, Helicity formalism for spin-2 particles, JHEP 09 (2003) 001 [hep-ph/0306182] [INSPIRE]. 
[23] A. Datta, E. Gabrielli and B. Mele, More on violation of angular-momentum selection rules in quantum gravity, hep-ph/0406149 [INSPIRE].

[24] R. Koekoek and R.F. Swarttouw, The Askey-scheme of hypergeometric orthogonal polynomials and its q-analogue, math/9602214.

[25] R.J. Eden, High energy collisions of elementary particles, Cambridge Univ. Press, Cambridge U.K. (1967).

[26] P. Collins and E.J. Squires, Regge poles in particle physics, Springer, Germany (1968).

[27] D. Ponomarev and A.A. Tseytlin, On quantum corrections in higher-spin theory in flat space, JHEP 05 (2016) 184 [arXiv: 1603.06273] [INSPIRE].

[28] E.S. Fradkin and A.A. Tseytlin, Renormalizable asymptotically free quantum theory of gravity, Nucl. Phys. B 201 (1982) 469 [inSPIRE].

[29] E.S. Fradkin and A.A. Tseytlin, One loop $\beta$-function in conformal supergravities, Nucl. Phys. B 203 (1982) 157 [INSPIRE].

[30] S.C. Lee and P. van Nieuwenhuizen, Counting of states in higher derivative field theories, Phys. Rev. D 26 (1982) 934 [INSPIRE].

[31] R.J. Riegert, The particle content of linearized conformal gravity, Phys. Lett. A 105 (1984) 110 [INSPIRE].

[32] J. Gustavsson, Some sums of Legendre and Jacobi polynomials, Math. Bohemica 126 (2001) 141.

[33] P. Donà, S. Giaccari, L. Modesto, L. Rachwal and Y. Zhu, Scattering amplitudes in super-renormalizable gravity, JHEP 08 (2015) 038 [arXiv: 1506.04589] [INSPIRE].

[34] K.S. Stelle, Classical gravity with higher derivatives, Gen. Rel. Grav. 9 (1978) 353 [INSPIRE].

[35] L. Dolan and J.N. Ihry, Conformal supergravity tree amplitudes from open twistor string theory, Nucl. Phys. B 819 (2009) 375 [arXiv:0811.1341] [INSPIRE].

[36] N. Berkovits and E. Witten, Conformal supergravity in twistor-string theory, JHEP 08 (2004) 009 [hep-th/0406051] [INSPIRE].

[37] T. Adamo and L. Mason, Twistor-strings and gravity tree amplitudes, Class. Quant. Grav. 30 (2013) 075020 [arXiv:1207.3602] [INSPIRE].

[38] T. Adamo and L. Mason, Conformal and Einstein gravity from twistor actions, Class. Quant. Grav. 31 (2014) 045014 [arXiv:1307.5043] [inSPIRE].

[39] J. Maldacena, Einstein gravity from conformal gravity, arXiv:1105.5632 [INSPIRE].

[40] J. Maldacena and A. Zhiboedov, Constraining conformal field theories with a higher spin symmetry, J. Phys. A 46 (2013) 214011 [arXiv:1112.1016] [INSPIRE].

[41] N. Boulanger, D. Ponomarev, E.D. Skvortsov and M. Taronna, On the uniqueness of higher-spin symmetries in AdS and CFT, Int. J. Mod. Phys. A 28 (2013) 1350162 [arXiv: 1305.5180] [INSPIRE].

[42] Y.S. Stanev, Constraining conformal field theory with higher spin symmetry in four dimensions, Nucl. Phys. B 876 (2013) 651 [arXiv:1307.5209] [INSPIRE].

[43] V. Alba and K. Diab, Constraining conformal field theories with a higher spin symmetry in $d>3$ dimensions, JHEP 03 (2016) 044 [arXiv: 1510.02535] [INSPIRE]. 